\title{
Doubly virtual Compton scattering and the beam normal spin asymmetry
}

\author{
Mikhail Gorchtein* \\ California Institute of Technology, Pasadena, California 91125, USA
}

(Received 7 December 2005; published 27 March 2006)

\begin{abstract}
We construct an invariant basis for Compton scattering with two virtual photons (VVCS). The basis tensors are chosen to be gauge invariant and orthogonal to each other. The properties of the corresponding 18 invariant amplitudes are studied in detail. We consider the special case of elastic VVCS with the virtualities of the initial and final photons equal. The invariant basis for VVCS in this orthogonal form does not exist in the literature. We furthermore use this VVCS tensor for a calculation of the beam normal spin asymmetry in the forward kinematics. For this, we relate the invariant amplitudes to the helicity amplitudes of the VVCS reaction. The imaginary parts of the latter are related to the inclusive cross section by means of the optical theorem. We use the phenomenological value of the transverse cross section $\sigma_{T} \sim 0.1 \mathrm{mb}$ and the Callan-Gross relation which relates the longitudinal cross section $\sigma_{L}$ to the transverse one. The result of the calculation agrees with an existing calculation, and predicts negative values of the asymmetry $B_{n}$ of order of $5 \mathrm{ppm}$ in the energy range from 3 to $45 \mathrm{GeV}$ and for very forward angles.
\end{abstract}

DOI: 10.1103/PhysRevC.73.035213

PACS number(s): 12.40.Nn, 13.40.Gp, 13.60.Fz, 13.60.Hb

\section{INTRODUCTION}

Over recent years, interest in Compton scattering with two virtual (typically, spacelike) photons has arisen and has been constantly growing. This interest is based on significant progress in precision electron-proton scatering experimental technique. Polarization transfer data for elastic electronproton scattering [1] showed that the extracted values of the electromagnetic form factors obtained with this technique differ significantly from the Rosenbluth separation data [2]. It has been pointed out that these two data sets may be reconciled by the proper inclusion of higher order contributions in the electromagnetic coupling constant $\alpha_{e m}$, namely the two photon exchange contribution [3]. Several model calculations using the elastic form factors only [4], the $\Delta(1232)$ resonance [5], and the partonic picture [6] show that the contribution of the two photon exchange exhibits the correct trend which may be enough to explain the discrepancy of the experimental results. However, a different class of observables has become experimentally accessible in the framework of parity-violating electron-proton scattering. These new observables involve the spin orientation normal to the reaction plane and have been shown to be directly related to the imaginary part of the elastic electron-proton scattering amplitude. Because one photon exchange does not lead to an imaginary part, the leading order contribution comes from the exchange of at least two photons [7]. Because the exchange of three or more photons is suppressed by powers of the small coupling constant $\alpha_{e} m$, a measurement of such an observable probably provides the cleanest way to access Compton scattering with two virtual photons. At present, several experimental data points exist in different kinematical regimes [8]. On the theoretical side, significant progress has been made toward quantitative understanding of the experimental data [9].

*Electronic addrsss: gorshtey@caltech.edu
These experimental and theoretical studies require a description of Compton scattering with two virtual photons. The general form of the explicitly gauge invariant basis for VVCS was developed in the 1970s by Tarrach [10]. The invariant amplitudes of Tarrach have the advantage that they do not have kinematical singularities nor constraints and thus can be used for a dispersion calculation. However, they were never used for a realistic calculation. One of the reasons is that, at that time, the VVCS reaction was of pure theoretical interest (apart from its forward limit that is used in DIS and described by the nucleon structure functions rather than the invariant amplitudes of Tarrach). The other reason is the technical difficulty in using this basis in a real calculation, which is because of the nonorthogonality of the basis tensors. Another approach was used by Prange for real Compton scattering [11] and then generalized to Compton scattering with a virtual initial photon by Berg and Lindner [12]. This method consists in defining the invariant Compton basis in terms of gauge-invariant basis tensors that are orthogonal to each other. In this work, we generalize this approach to the case when the outgoing photon is also virtual. In Sec. II, we introduce the VVCS tensor in such a form, and study the properties of the invariant amplitudes. We also consider the special case of elastic VVCS with initial and outgoing photons of equal virtuality. In the literature, the invariant Compton tensor for this reaction exists only for the forward case. We obtain here the result for the general case. In Sec. III, we use the introduced from of the Compton tensor for a practical calculation of the beam normal spin asymmetry $B_{n}$ in the forward kinematics.

\section{DOUBLY VCS TENSOR BASIS}

In this section, the doubly virtual Compton scattering (VVCS) tensor will be introduced and the properties of the invariant VVCS amplitudes will be investigated. We start by reviewing existing developments on invariant Compton tensors. A general Lorentz and gauge-invariant VVCS tensor 
was constructed by Tarrach [10] with the corresponding amplitudes free from kinematical singularities and constraints. However, it appears problematic to use this tensor basis for practical calculations. Therefore, we turn to the method used by Prange for RCS [11] and extended to VCS (with only one virtual photon) by Berg and Lindner [12]. We consider a scattering process $\gamma^{*}\left(q_{1}\right)+N(p) \rightarrow \gamma^{*}\left(q_{2}\right)+N\left(p^{\prime}\right)$ and define the four-vectors:

$$
\begin{aligned}
P & =\frac{p+p^{\prime}}{2} \\
\tilde{K} & =\frac{q_{1}+q_{2}}{2} \\
L & =\frac{q_{1}-q_{2}}{2}=\frac{p^{\prime}-p}{2} .
\end{aligned}
$$

The method of Ref. [11] for RCS amounts to defining two orthogonal vectors,

$$
\begin{aligned}
P^{\prime \mu} & =P^{\mu}-\frac{P \cdot \tilde{K}}{\tilde{K}^{2}} \tilde{K}^{\mu}, \\
n^{\mu} & =\varepsilon^{\mu \alpha \beta \gamma} P_{\alpha}^{\prime} \tilde{K}_{\beta} L_{\gamma},
\end{aligned}
$$

where we use the convention $\varepsilon^{0123}=-1$. The four vectors $P^{\prime}, n, L^{\prime}$, and $\tilde{K}$ form an orthogonal basis. Of these vectors, orthogonal and explicitly gauge-invariant tensors can be constructed. The six independent Lorentz-invariant structures that are needed to describe RCS in the most general case are [11]:

$$
\begin{aligned}
M_{\mathrm{RCS}}^{\mu \nu}= & \bar{N}^{\prime}\left\{\frac{P^{\prime \mu} P^{\prime \nu}}{P^{\prime 2}}\left(B_{1}+\tilde{K} B_{2}\right)+\frac{n^{\mu} n^{\nu}}{n^{2}}\left(B_{3}+\tilde{K} B_{4}\right)\right. \\
& +\frac{P^{\prime \mu} n^{\nu}-n^{\mu} P^{\prime \nu}}{P^{\prime 2} n^{2}} i \gamma_{5} B_{7} \\
& \left.+\frac{P^{\prime \mu} n^{\nu}+n^{\mu} P^{\prime \nu}}{P^{\prime 2} n^{2}} \not h B_{6}\right\} N .
\end{aligned}
$$

We note that the terms $\sim\left(P^{\prime \mu} n^{\nu}+P^{\prime \nu} n^{\mu}\right) / P^{\prime 2} n^{2} i \gamma_{5}$ and $\left(P^{\prime \mu} n^{\nu}-P^{\prime \nu} n^{\mu}\right) / P^{\prime 2} n^{2} \not h$ are ruled out by time-reversal invariance. If we allow for one of the photons (normally, the initial one) to be virtual, this restriction is relaxed because the initial and final states are not identical anymore. The method should be modified as follows [12]: because the condition $L \cdot \tilde{K}=0$ is no longer valid, we introduce

$$
\begin{aligned}
L^{\prime \mu} & =L^{\mu}-\frac{L \cdot \tilde{K}}{\tilde{K}^{2}} \tilde{K}^{\mu}, \\
P^{\prime \mu} & =P^{\mu}-\frac{P \cdot \tilde{K}}{\tilde{K}^{2}} \tilde{K}^{\mu}-\frac{P \cdot L^{\prime}}{L^{\prime 2}} L^{\prime \mu}, \\
n^{\mu} & =\varepsilon^{\mu \alpha \beta \gamma} P_{\alpha}^{\prime} \tilde{K}_{\beta} L_{\gamma}^{\prime},
\end{aligned}
$$

which are orthogonal to each other and to the vector $\tilde{K}$. Furthermore, to take into account the longitudinal polarization of the virtual photon, a gauge-invariant vector is introduced,

$$
\tilde{K}^{\prime \mu}=\tilde{K}^{\mu}-\frac{q_{1} \cdot \tilde{K}}{q_{1} \cdot L^{\prime}} L^{\prime \mu},
$$

which obeys $q_{1} \cdot \tilde{K}^{\prime}=0$. With these modifications, the most general VCS tensor basis takes the form [12]:

$$
\begin{aligned}
M_{\mathrm{VCS}}^{\mu \nu}= & \bar{N}^{\prime}\left\{\frac{P^{\prime \mu} P^{\prime \nu}}{P^{\prime 2}}\left(B_{1}+\tilde{K} B_{2}\right)+\frac{n^{\mu} n^{\nu}}{n^{2}}\left(B_{3}+\tilde{K} B_{4}\right)\right. \\
& +\frac{P^{\prime \mu} n^{\nu}+n^{\mu} P^{\prime \nu}}{P^{\prime 2} n^{2}}\left(i \gamma_{5} B_{5}+\not h B_{6}\right) \\
& +\frac{P^{\prime \mu} n^{\nu}-n^{\mu} P^{\prime \nu}}{P^{\prime 2} n^{2}}\left(i \gamma_{5} B_{7}+\not h B_{8}\right) \\
& +\frac{\tilde{K}^{\prime \mu} P^{\prime \nu}}{P^{\prime 2} \tilde{K}^{2}}\left(B_{9}+\tilde{K} B_{10}\right) \\
& \left.+\frac{\tilde{K}^{\prime \mu} n^{\nu}}{n^{2} \tilde{K}^{2}}\left(i \gamma_{5} B_{11}+\not h B_{12}\right)\right\} N
\end{aligned}
$$

The Compton tensor in this form, for the case where both photons are virtual does not exist in the literature. We follow here the scheme proposed in Ref. [12] and generalize the invariant Compton tensor for the VVCS case. For this, we introduce another gauge-invariant vector with respect to the outgoing virtual photon $q_{2}$,

$$
\tilde{K}^{\prime \prime \nu}=\tilde{K}^{\nu}-\frac{q_{2} \cdot \tilde{K}}{q_{2} \cdot L^{\prime}} L^{\prime \nu} .
$$

We finally obtain the 18 different structures that contain all the information about Compton scattering with two virtual photons:

$$
\begin{aligned}
M_{\mathrm{VVCS}}^{\mu \nu}= & \bar{N}^{\prime}\left\{\frac{P^{\prime \mu} P^{\prime \nu}}{P^{\prime 2}}\left(B_{1}+\tilde{K} B_{2}\right)+\frac{n^{\mu} n^{\nu}}{n^{2}}\left(B_{3}+\tilde{K} B_{4}\right)\right. \\
& +\frac{P^{\prime \mu} n^{\nu}+n^{\mu} P^{\prime \nu}}{P^{\prime 2} n^{2}}\left(i \gamma_{5} B_{5}+\not h B_{6}\right) \\
& +\frac{P^{\prime \mu} n^{\nu}-n^{\mu} P^{\prime \nu}}{P^{\prime 2} n^{2}}\left(i \gamma_{5} B_{7}+\not h B_{8}\right) \\
& +\frac{\tilde{K}^{\prime \mu} P^{\prime \nu}}{P^{\prime 2} \tilde{K}^{2}}\left(B_{9}+\tilde{K} B_{10}\right) \\
& +\frac{\tilde{K}^{\prime \mu} n^{\nu}}{n^{2} \tilde{K}^{2}}\left(i \gamma_{5} B_{11}+\not h B_{12}\right) \\
& +\frac{P^{\prime \mu} \tilde{K}^{\prime \prime \nu}}{P^{\prime 2} \tilde{K}^{2}}\left(B_{13}+\tilde{K} B_{14}\right) \\
& +\frac{n^{\mu} \tilde{K}^{\prime \prime \nu}}{n^{2} \tilde{K}^{2}}\left(i \gamma_{5} B_{15}+\not h B_{16}\right) \\
& \left.+\frac{\tilde{K}^{\prime \mu} \tilde{K}^{\prime \prime \nu}}{\tilde{K}^{2}}\left(B_{17}+\tilde{K} B_{18}\right)\right\} N .
\end{aligned}
$$

The invariant amplitudes $B_{i}$ are functions of the invariants:

$$
B_{i}=B_{i}\left(q_{1}^{2}, q_{2}^{2}, q_{1} \cdot q_{2}, P \cdot \tilde{K}\right) .
$$

We next consider the behavior of the invariant amplitudes under photon and nucleon crossing.

\section{A. Nucleon crossing}

This transformation is a combination of partial parity transformation and charge conjugation: $\left[\gamma^{*}\left(q_{1}\right)+N(p) \rightarrow\right.$ $\left.\gamma^{*}\left(q_{2}\right)+N\left(p^{\prime}\right)\right] \rightarrow\left[\gamma^{*}\left(q_{1}\right)+\bar{N}\left(-p^{\prime}\right) \rightarrow \gamma^{*}\left(q_{2}\right)+\bar{N}(-p)\right]$. We note that this is an unphysical transformation because only the hadronic part of the kinematics is transformed, whereas 
the photons remain unchanged. This transformation, however, relates two reactions that should have the same structure. Under nucleon crossing, we have:

$$
\begin{aligned}
P & \rightarrow-P, \\
n & \rightarrow-n, \\
\tilde{K} & \rightarrow \tilde{K}, \\
L^{\prime} & \rightarrow L^{\prime}, \\
\tilde{K}^{\prime} & \rightarrow \tilde{K}^{\prime}, \\
\tilde{K}^{\prime \prime} & \rightarrow \tilde{K}^{\prime \prime} \\
\tilde{K} & \rightarrow \mathcal{C}^{\dagger} \tilde{K} \mathcal{C}=-\tilde{K}, \\
\not h & \rightarrow-\mathcal{C}^{\dagger} \not h \mathcal{C}=\not h, \\
\gamma_{5} & \rightarrow \mathcal{C}^{\dagger} \gamma_{5} \mathcal{C}=\gamma_{5} .
\end{aligned}
$$

Hence, nucleon crossing transforms the VVCS tensor as

$$
\begin{aligned}
{ }^{N} \tilde{M}_{\mathrm{VVCS}}^{\mu \nu}= & \bar{N}\left\{\frac{P^{\prime \mu} P^{\prime \nu}}{P^{\prime 2}}\left({ }^{N} \tilde{B}_{1}-\tilde{K}^{N} \tilde{B}_{2}\right)\right. \\
& +\frac{n^{\mu} n^{\nu}}{n^{2}}\left({ }^{N} \tilde{B}_{3}-\tilde{K}^{N} \tilde{B}_{4}\right) \\
& +\frac{P^{\prime \mu} n^{\nu}+n^{\mu} P^{\prime \nu}}{P^{\prime 2} n^{2}}\left(i \gamma_{5}{ }^{N} \tilde{B}_{5}+\not h^{N} \tilde{B}_{6}\right) \\
& +\frac{P^{\prime \mu} n^{\nu}-n^{\mu} P^{\prime \nu}}{P^{\prime 2} n^{2}}\left(i \gamma_{5}{ }^{N} \tilde{B}_{7}+\not h^{N} \tilde{B}_{8}\right) \\
& -\frac{\tilde{K}^{\prime \mu} P^{\prime \nu}}{P^{\prime 2} \tilde{K}^{2}}\left({ }^{N} \tilde{B}_{9}-\tilde{K}^{N} \tilde{B}_{10}\right) \\
& -\frac{\tilde{K}^{\prime \mu} n^{\nu}}{n^{2} \tilde{K}^{2}}\left(i \gamma_{5}{ }^{N} \tilde{B}_{11}+\not h^{N} \tilde{B}_{12}\right) \\
& -\frac{P^{\prime \mu} \tilde{K}^{\prime \prime \nu}}{P^{\prime 2} \tilde{K}^{2}}\left({ }^{N} \tilde{B}_{13}-\not \tilde{K}^{N} \tilde{B}_{14}\right) \\
& -\frac{n^{\mu} \tilde{K}^{\prime \prime \nu}}{n^{2} \tilde{K}^{2}}\left(i \gamma_{5}{ }^{N} \tilde{B}_{15}+\not h^{N} \tilde{B}_{16}\right) \\
& \left.+\frac{\tilde{K}^{\prime \mu} \tilde{K}^{\prime \prime \nu}}{\tilde{K}^{2}}\left({ }^{N} \tilde{B}_{17}-\tilde{K}^{N} \tilde{B}_{18}\right)\right\} N^{\prime} .
\end{aligned}
$$

In the above equation, ${ }^{N} \tilde{B}_{i}$ stands for the nucleon crossing transform of the amplitude $B_{i}$. For the invariant amplitudes, nucleon crossing amounts to the substitution $P \cdot \tilde{K} \rightarrow-P$. $\tilde{K}$, whereas the other arguments remain unchanged. We next list the behavior of the invariant amplitudes $B_{i}$ under nucleon crossing:

$$
\begin{aligned}
B_{i}\left(q_{1}^{2}, q_{2}^{2}, q_{1} \cdot q_{2},-P \cdot \tilde{K}\right) & =+B_{i}\left(q_{1}^{2}, q_{2}^{2}, q_{1} \cdot q_{2}, P \cdot \tilde{K}\right) \\
\text { for } i & =1,3,5,6,7,8,10,14,17 \\
B_{i}\left(q_{1}^{2}, q_{2}^{2}, q_{1} \cdot q_{2},-P \cdot \tilde{K}\right) & =-B_{i}\left(q_{1}^{2}, q_{2}^{2}, q_{1} \cdot q_{2}, P \cdot \tilde{K}\right) \\
\text { for } i & =2,4,9,11,12,13,15,16,18 .
\end{aligned}
$$

\section{B. Photon crossing}

Photon crossing relates two reactions: $\left[\gamma^{*}\left(q_{1}\right)+N(p) \rightarrow\right.$ $\left.\gamma^{*}\left(q_{2}\right)+N\left(p^{\prime}\right)\right] \rightarrow\left[\gamma^{*}\left(-q_{2}\right)+N(p) \rightarrow \gamma^{*}\left(-q_{1}\right)+N\left(p^{\prime}\right)\right]$. In the general case of $q_{1}^{2} \neq q_{2}^{2}$, the Compton amplitude must not be invariant under photon crossing. It is, however, a relevant symmetry in the elastic case $q_{1}^{2}=q_{2}^{2}$. Under photon crossing we have:

$$
\begin{aligned}
& \tilde{K} \rightarrow-\tilde{K}, \\
& \mu \leftrightarrow v \\
& P \rightarrow P, \\
& P^{\prime} \rightarrow P^{\prime}, \\
& L^{\prime} \rightarrow L^{\prime}, \\
& n \rightarrow-n, \\
& \tilde{K}^{\prime} \rightarrow-\tilde{K}^{\prime \prime}, \\
& \tilde{K}^{\prime \prime} \rightarrow-\tilde{K}^{\prime} .
\end{aligned}
$$

Hence, photon crossing transforms the VVCS tensor as

$$
\begin{aligned}
\gamma^{\gamma} \tilde{M}_{\mathrm{VVCS}}^{\mu \nu}= & \bar{N}^{\prime}\left\{\frac{P^{\prime \mu} P^{\prime \nu}}{P^{\prime 2}}\left({ }^{\gamma} \tilde{B}_{1}-\tilde{K}^{\gamma} \tilde{B}_{2}\right)\right. \\
& +\frac{n^{\mu} n^{\nu}}{n^{2}}\left({ }^{\gamma} \tilde{B}_{3}-\tilde{K}^{\gamma} \tilde{B}_{4}\right) \\
& -\frac{P^{\prime \mu} n^{\nu}+n^{\mu} P^{\prime \nu}}{P^{\prime 2} n^{2}}\left(i \gamma_{5}{ }^{\gamma} \tilde{B}_{5}-\not h^{\gamma} \tilde{B}_{6}\right) \\
& +\frac{P^{\prime \mu} n^{\nu}-n^{\mu} P^{\prime \nu}}{P^{\prime 2} n^{2}}\left(i \gamma_{5}{ }^{\gamma} \tilde{B}_{7}-\not h^{\gamma} \tilde{B}_{8}\right) \\
& -\frac{P^{\prime \mu} \tilde{K}^{\prime \prime \nu}}{P^{\prime 2} \tilde{K}^{2}}\left({ }^{\gamma} \tilde{B}_{9}-\tilde{K}^{\gamma} \tilde{B}_{10}\right) \\
& +\frac{n^{\mu} \tilde{K}^{\prime \prime \nu}}{n^{2} \tilde{K}^{2}}\left(i \gamma_{5}{ }^{\gamma} \tilde{B}_{11}+\not h^{\gamma} \tilde{B}_{12}\right) \\
& -\frac{\tilde{K}^{\prime \mu} P^{\prime \nu}}{P^{\prime 2} \tilde{K}^{2}}\left({ }^{\gamma} \tilde{B}_{13}-\tilde{K}^{\gamma} \tilde{B}_{14}\right) \\
& +\frac{\tilde{K}^{\prime \mu} n^{\nu}}{n^{2} \tilde{K}^{2}}\left(i \gamma_{5}{ }^{\gamma} \tilde{B}_{15}+\not h^{\gamma} \tilde{B}_{16}\right) \\
& \left.+\frac{\tilde{K}^{\prime \mu} \tilde{K}^{\prime \prime \nu}}{\tilde{K}^{2}}\left({ }^{\gamma} \tilde{B}_{17}-\tilde{K}^{\gamma} \tilde{B}_{18}\right)\right\} N^{\prime} .
\end{aligned}
$$

In the above equation, ${ }^{\gamma} \tilde{B}_{i}$ stands for the nucleon crossing transform of the amplitude $B_{i}$. Apart from the substitution $P$. $\tilde{K} \rightarrow-P \cdot \tilde{K}$, photon crossing interchanges the virtualities of the photons as arguments of the invariant amplitudes, $q_{1}^{2} \leftrightarrow q_{2}^{2}$. We find following behavior under photon crossing:

$$
\begin{aligned}
B_{i}\left(q_{2}^{2}, q_{1}^{2}, q_{1} \cdot q_{2},-P \cdot \tilde{K}\right) & =+B_{i}\left(q_{1}^{2}, q_{2}^{2}, q_{1} \cdot q_{2}, P \cdot \tilde{K}\right) \\
\text { for } i & =1,3,6,7,17 \\
B_{i}\left(q_{2}^{2}, q_{1}^{2}, q_{1} \cdot q_{2},-P \cdot \tilde{K}\right) & =-B_{i}\left(q_{1}^{2}, q_{2}^{2}, q_{1} \cdot q_{2}, P \cdot \tilde{K}\right) \\
\text { for } i & =2,4,5,8,18 \\
B_{9}\left(q_{2}^{2}, q_{1}^{2}, q_{1} \cdot q_{2},-P \cdot \tilde{K}\right) & =-B_{13}\left(q_{1}^{2}, q_{2}^{2}, q_{1} \cdot q_{2}, P \cdot \tilde{K}\right) \\
B_{10}\left(q_{2}^{2}, q_{1}^{2}, q_{1} \cdot q_{2},-P \cdot \tilde{K}\right) & =B_{14}\left(q_{1}^{2}, q_{2}^{2}, q_{1} \cdot q_{2}, P \cdot \tilde{K}\right) \\
B_{13}\left(q_{2}^{2}, q_{1}^{2}, q_{1} \cdot q_{2},-P \cdot \tilde{K}\right) & =-B_{9}\left(q_{1}^{2}, q_{2}^{2}, q_{1} \cdot q_{2}, P \cdot \tilde{K}\right) \\
B_{14}\left(q_{2}^{2}, q_{1}^{2}, q_{1} \cdot q_{2},-P \cdot \tilde{K}\right) & =B_{10}\left(q_{1}^{2}, q_{2}^{2}, q_{1} \cdot q_{2}, P \cdot \tilde{K}\right) \\
B_{11}\left(q_{2}^{2}, q_{1}^{2}, q_{1} \cdot q_{2},-P \cdot \tilde{K}\right) & =B_{15}\left(q_{1}^{2}, q_{2}^{2}, q_{1} \cdot q_{2}, P \cdot \tilde{K}\right) \\
B_{12}\left(q_{2}^{2}, q_{1}^{2}, q_{1} \cdot q_{2},-P \cdot \tilde{K}\right) & =-B_{16}\left(q_{1}^{2}, q_{2}^{2}, q_{1} \cdot q_{2}, P \cdot \tilde{K}\right) \\
B_{15}\left(q_{2}^{2}, q_{1}^{2}, q_{1} \cdot q_{2},-P \cdot \tilde{K}\right) & =B_{11}\left(q_{1}^{2}, q_{2}^{2}, q_{1} \cdot q_{2}, P \cdot \tilde{K}\right) \\
B_{16}\left(q_{2}^{2}, q_{1}^{2}, q_{1} \cdot q_{2},-P \cdot \tilde{K}\right) & =-B_{12}\left(q_{1}^{2}, q_{2}^{2}, q_{1} \cdot q_{2}, P \cdot \tilde{K}\right) .
\end{aligned}
$$


We now combine photon and nucleon crossing, which corresponds to applying a CP-transformation to the full reaction. Combining Eqs. (12) and (15), we obtain

$$
\begin{aligned}
& B_{5,8}, B_{9}-B_{13}, \quad B_{10}-B_{14}, \\
& B_{11}+B_{15}, \quad B_{12}+B_{16} \sim\left(q_{1}^{2}-q_{2}^{2}\right)^{2 n+1},
\end{aligned}
$$

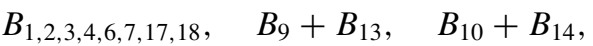

$$
\begin{aligned}
& B_{11}-B_{15}, \quad B_{12}-B_{16} \sim\left(q_{1}^{2} \pm q_{2}^{2}\right)^{2 n},
\end{aligned}
$$

with $n=0,1,2, \ldots 1$

As a consequence, the VVCS tensor simplifies in the elastic case (EVVCS), $q_{1}^{2}=q_{2}^{2}$ :

$$
\begin{aligned}
M_{\mathrm{EVVCS}}^{\mu \nu}= & \bar{N}^{\prime}\left\{\frac{P^{\prime \mu} P^{\prime \nu}}{P^{\prime 2}}\left(B_{1}+\tilde{K} B_{2}\right)+\frac{n^{\mu} n^{\nu}}{n^{2}}\left(B_{3}+\tilde{K} B_{4}\right)\right. \\
& +\frac{P^{\prime \mu} n^{\nu}+n^{\mu} P^{\prime \nu}}{P^{\prime 2} n^{2}} \not h B_{6}+\frac{P^{\prime \mu} n^{\nu}-n^{\mu} P^{\prime \nu}}{P^{\prime 2} n^{2}} i \gamma_{5} B_{7} \\
& +\frac{\tilde{K}^{\prime \mu} P^{\prime \nu}+P^{\prime \mu} \tilde{K}^{\prime \prime \nu}}{P^{\prime 2} \tilde{K}^{2}}\left(B_{9}+\tilde{K} B_{10}\right) \\
& +\frac{\tilde{K}^{\prime \mu} n^{\nu}-n^{\mu} \tilde{K}^{\prime \prime \nu}}{n^{2} \tilde{K}^{2}} i \gamma_{5} B_{11}+\frac{\tilde{K}^{\prime \mu} n^{\nu}+n^{\mu} \tilde{K}^{\prime \prime \nu}}{n^{2} \tilde{K}^{2}} \not h B_{12} \\
& \left.+\frac{\tilde{K}^{\prime \mu} \tilde{K}^{\prime \prime \nu}}{\tilde{K}^{2}}\left(B_{17}+\tilde{K} B_{18}\right)\right\} N .
\end{aligned}
$$

We finally investigate the RCS limit, i.e., let the virtualities of the photons go to zero. In this case,

$$
\begin{aligned}
\tilde{K}^{\prime \mu} & \rightarrow q_{1}^{\mu} \\
\tilde{K}^{\prime \prime \nu} & \rightarrow q_{2}^{\nu} .
\end{aligned}
$$

In an observable, the Compton tensor is contracted with the photon polarization vectors, $\varepsilon_{\lambda}\left(q_{1}\right) \cdot \tilde{K}^{\prime} \rightarrow \varepsilon_{\lambda}\left(q_{1}\right) \cdot q_{1}=0$. Though the amplitudes $B_{9,10,11,12,17,18}$ should not necessarily vanish in this limit, the corresponding terms do not contribute to any observables. Therefore, at the real photon point we recover the old RCS tensor of Prange of Eq. (3).

It should be stressed that doubly VCS tensors for inelastic $\left(q_{1}^{2} \neq q_{2}^{2}\right)$ and elastic $\left(q_{1}^{2}=q_{2}^{2}\right)$ cases in the form of Eqs. (8) and (17) do not exist in the literature.

\section{APPLICATION: BEAM NORMAL SPIN ASYMMETRY}

We consider elastic electron-proton scattering $e^{-}(k)+$ $N(p) \rightarrow e^{-}\left(k^{\prime}\right)+N\left(p^{\prime}\right)$, with the electron beam polarized normally to the scattering plane with the polarization vector defined as

$$
\xi^{\mu}=\left(0, \frac{\vec{k} \times \vec{k}^{\prime}}{\left|\vec{k} \times \vec{k}^{\prime}\right|}\right)
$$

The single-spin asymmetry defined as $B_{n}=$ $\left(\sigma_{\uparrow}-\sigma_{\downarrow}\right) /\left(\sigma_{\uparrow}+\sigma_{\downarrow}\right)$ is refered to as beam normal spin asymmetry. It was shown that such an asymmetry, to leading

\footnotetext{
${ }^{1}$ We do not expect any negative powers of $q_{1}^{2}-q_{2}^{2}$ because it would lead to an unphysical singularity.
}

order in the electromagnetic coupling constant $\alpha_{e m} \approx 1 / 137$, is given by

$$
B_{n}=\frac{\sum_{\text {spins }} 2 \operatorname{Im}\left(\mathcal{M}_{1 \gamma}^{*} \operatorname{Abs} \mathcal{M}_{2 \gamma}\right)}{\sum_{\text {spins }}\left|\mathcal{M}_{1 \gamma}\right|^{2}} .
$$

The absorptive part of the two-photon exchange (TPE) graph is given by

$$
\begin{aligned}
\operatorname{Abs} \mathcal{M}_{2 \gamma}= & e^{4} \int \frac{\left|\vec{k}_{1}\right|^{2} d\left|\vec{k}_{1}\right| d \Omega_{k_{1}}}{2 E_{1}(2 \pi)^{3}} \bar{u}^{\prime} \gamma_{\nu}\left(k_{1}+m\right) \gamma_{\mu} u \\
& \times \frac{1}{Q_{1}^{2} Q_{2}^{2}} \operatorname{Abs} M^{\mu \nu}\left(w^{2}, t, Q_{1}^{2}, Q_{2}^{2}\right),
\end{aligned}
$$

where $M^{\mu v}$ is the VVCS tensor. Furthermore, the asymmetry can be written as

$$
B_{n}=\frac{e^{2} t}{D(s, t)} \int \frac{\left|\vec{k}_{1}\right|^{2} d\left|\vec{k}_{1}\right| d \Omega_{k_{1}}}{2 E_{1}(2 \pi)^{3}} \frac{1}{Q_{1}^{2} Q_{2}^{2}} \operatorname{Im} L_{\alpha \mu \nu} H^{\alpha \mu \nu} .
$$

The leptonic tensor $L_{\alpha \mu \nu}$ is given by

$$
L_{\alpha \mu \nu}=\operatorname{Tr}\left(k^{\prime}+m\right) \gamma_{\nu}\left(k_{1}+m\right) \gamma_{\mu} \gamma_{5} \xi(k+m) \gamma_{\alpha},
$$

whereas the hadronic tensor is defined as

$$
H^{\alpha \mu \nu}=\sum_{\text {spins }} \operatorname{Abs} M^{\mu \nu}\left(\bar{N}^{\prime} \Gamma^{\alpha} N\right)^{*},
$$

with $\Gamma^{\alpha}=G_{M}(-t) \gamma^{\alpha}-F_{2}(-t) \frac{P^{\alpha}}{M}$. In the following, we use the hadronic tensor in the form introduced in the previous section for the most general inelastic case. The structure of the tensor of Eq. (8) can be represented as

$$
\begin{aligned}
M^{\mu \nu}= & A^{\mu \nu} \cdot \bar{N}^{\prime} N+B^{\mu \nu} \cdot \bar{N}^{\prime} \tilde{K} N \\
& +C^{\mu \nu} \cdot \bar{N}^{\prime} i \gamma_{5} N+D^{\mu \nu} \cdot \bar{N}^{\prime} \not h N .
\end{aligned}
$$

The tensors $(A, B, C, D)^{\mu v}$ are defined in the appendix. The sum over spins in the hadronic tensor can be readily performed with the result:

$$
\begin{aligned}
H^{\alpha \mu \nu}= & 8 M P^{\alpha}\left[G_{E} A^{\mu \nu}+\frac{P \tilde{K}}{M} F_{1} B^{\mu \nu}\right] \\
& +8 L^{2} G_{M}\left[\left(\tilde{K}^{\alpha}-\frac{L \tilde{K}}{L^{2}} L^{\alpha}\right) B^{\mu \nu}+n^{\alpha} D^{\mu \nu}\right] .
\end{aligned}
$$

Notice that the tensor $C^{\mu v}$ appears with $\gamma_{5}$, and does not contribute to this observable. We list here the result of the tensor contraction and refer the reader to the appendix for the details of the calculation:

$$
\begin{aligned}
& L_{\alpha \mu \nu} H^{\alpha \mu \nu}=64 \operatorname{imM}(n \cdot \xi) \\
& \quad \times\left\{G_{E}\left[B_{1}+B_{3}-\frac{L^{2} Q_{1}^{2} Q_{2}^{2}}{\tilde{K}^{2}\left(L^{\prime 2}\right)^{2}} B_{17}\right]\right. \\
& +\frac{P \tilde{K}}{M} F_{1}\left[B_{2}+B_{4}-\frac{L^{2} Q_{1}^{2} Q_{2}^{2}}{\tilde{K}^{2}\left(L^{\prime 2}\right)^{2}} B_{18}\right] \\
& -\frac{2\left(P^{\prime} k_{1}\right)(P \tilde{K})}{P^{\prime 2} \tilde{K}^{2}}\left(G_{E} B_{1}+\frac{P \tilde{K}}{M} F_{1} B_{2}\right) \\
& +\frac{Q_{1}^{2}}{n^{2}}\left[P K-P \tilde{K} \frac{\left(q_{2} L\right) L^{2}}{\tilde{K}^{2} L^{\prime 2}}\right]\left(G_{E} B_{9}+\frac{P \tilde{K}}{M} F_{1} B_{10}\right)
\end{aligned}
$$




$$
\begin{aligned}
& \left.+\frac{Q_{2}^{2}}{n^{2}}\left[P K+P \tilde{K} \frac{\left(q_{1} L\right) L^{2}}{\tilde{K}^{2} L^{\prime 2}}\right]\left(G_{E} B_{13}+\frac{P \tilde{K}}{M} F_{1} B_{14}\right)\right\} \\
& +128 i m L^{2} G_{M} \frac{(n \xi)}{P^{\prime 2}}\left[B_{8}-\left(P^{\prime} K\right) B_{2}\right. \\
& \left.+\frac{Q_{1}^{2}+Q_{2}^{2}}{16 \tilde{K}^{2} L^{\prime 2}}\left(Q_{1}^{2} B_{10}+Q_{2}^{2} B_{14}\right)\right]-128 i m L^{2} \frac{\left(n k_{1}\right)\left(k_{1} \xi\right)}{n^{2}} \\
& \times\left\{M^{3} G_{E}\left[\left(1-\frac{t}{4 M^{2}}\right) B_{3}+\frac{P \tilde{K}}{M} B_{4}\right]-L^{2} G_{M} B_{6}\right. \\
& \left.+G_{M}\left[(L \tilde{K}) B_{8}+\frac{(P \tilde{K}) L^{2}}{2 \tilde{K}^{2} L^{\prime 2}}\left(Q_{1}^{2} B_{12}+Q_{2}^{2} B_{16}\right)\right]\right\} .
\end{aligned}
$$

For forward kinematics, the virtualities of the photons are very close to each other such that the contributions of the terms $\sim\left(Q_{1}^{2}-Q_{2}^{2}\right)^{n}$ are suppressed by powers of $t / s$. In this case, we can use realtions between the amplitudes $B_{i}$ established in the previous sections. Denoting $Q_{1}^{2}=Q_{2}^{2} \equiv Q^{2}$, we obtain:

$$
\begin{aligned}
L_{\alpha \mu \nu} & H_{\mathrm{EVVCS}}^{\alpha \mu \nu} \\
= & 64 \operatorname{im} M(n \cdot \xi)\left\{G_{E}\left(B_{1}+B_{3}-\frac{Q^{4}}{\tilde{K}^{2} L^{\prime 2}} B_{17}\right)\right. \\
& +\frac{P \tilde{K}}{M} F_{1}\left(B_{2}+B_{4}-\frac{Q^{4}}{\tilde{K}^{2} L^{\prime 2}} B_{18}\right) \\
& -\frac{2\left(P^{\prime} k_{1}\right)(P \tilde{K})}{P^{\prime 2} \tilde{K}^{2}}\left(G_{E} B_{1}+\frac{P \tilde{K}}{M} F_{1} B_{2}\right) \\
& \left.+\frac{2 Q^{2}}{n^{2}}\left(P K+P \tilde{K} \frac{L^{2}}{\tilde{K}^{2}}\right)\left(G_{E} B_{9}+\frac{P \tilde{K}}{M} F_{1} B_{10}\right)\right\} \\
& -128 i m L^{2} G_{M}(n \xi)\left[\frac{\left(P^{\prime} K\right)}{P^{\prime 2}} B_{2}+\frac{Q^{4}}{4 n^{2}} B_{10}\right] \\
& -128 i m L^{2} \frac{\left(n k_{1}\right)\left(k_{1} \xi\right)}{n^{2}} \\
& \times\left\{M^{3} G_{E}\left[\left(1-\frac{t}{4 M^{2}}\right) B_{3}+\frac{P \tilde{K}}{M} B_{4}\right]\right. \\
& \left.-G_{M}\left[L^{2} B_{6}-(P \tilde{K}) \frac{Q^{2}}{\tilde{K}^{2}} B_{12}\right]\right\} .
\end{aligned}
$$

A further simplification can be obtained in the quasi-RCS approximation. It amounts to considering a soft intermediate electron. This kinematical region is enhanced by large logarithms $\sim \ln \left(-t / m^{2}\right)$. This approximation corresponds to taking $k_{1}=0$ and $Q_{1}^{2}=Q_{2}^{2}=0$ in the numerator.

$$
\begin{aligned}
& L_{\alpha \mu \nu} H_{q \mathrm{RCS}}^{\alpha \mu \nu}=64 \operatorname{imM}(n \cdot \xi) \\
& \quad \times\left[G_{E}\left(B_{1}+B_{3}\right)+\frac{P \tilde{K}}{M} F_{1}\left(B_{2}+B_{4}\right)\right] .
\end{aligned}
$$

\section{CALCUlation OF $B_{n}$ IN THE FORWARD KINEMATICS}

In this section, we are going to apply the formalism developed in the previous section to a calculation with Regge kinematics. Recent calculations have shown that in the forward kinematics, a logarithmic enhancement takes place [13], but the contribution of the $\ln ^{2}\left(-t / m^{2}\right)$ term comes with photon helicity flip amplitude of RCS and its contribution is negligibly small $[14,15]$. Vanishing of the RCS contribution at the exact quasi-RCS point requires that we take into account the full $k_{1}$ and $Q_{1,2}^{2}$ dependence of the hadronic tensor. The only simplification that arises because of the forward kinematics is based on the observation that integrals containing powers of $\left(Q_{1}^{2}-Q_{2}^{2}\right)$ are suppressed by powers of the (small) momentum transfer $t$. It can be shown, in fact, that

$$
\begin{aligned}
& \int \frac{\left|\vec{k}_{1}\right|^{2} d\left|\vec{k}_{1}\right| d \Omega_{k_{1}}}{2 E_{1}(2 \pi)^{3}} \frac{Q_{1}^{2}-Q_{2}^{2}}{Q_{1}^{2} Q_{2}^{2}}=0, \\
& \int \frac{\left|\vec{k}_{1}\right|^{2} d\left|\vec{k}_{1}\right| d \Omega_{k_{1}}}{2 E_{1}(2 \pi)^{3}} \frac{\left(Q_{1}^{2}-Q_{2}^{2}\right)^{2}}{Q_{1}^{2} Q_{2}^{2}}=\mathcal{O}(t / s),
\end{aligned}
$$

and we refer the reader to the appendix for details of the calculation. Therefore, in the Regge regime, elastic VVCS should give the leading $t$ contribution, and we can use the hadronic tensor in the form of Eq. (17), whose contribution to $B_{n}$ is given by Eq. (28). To calculate the asymmetry $B_{n}$ in these kinematics, we are going to relate the invariant amplitudes $B_{i}$ to the helicity amplitudes of doubly VCS. The imaginary parts of these latter are related to the total photoabsorption cross section by means of the optical theorem.

\section{A. Helicity amplitudes of VVCS with $Q_{1}^{2}=Q_{2}^{2}$}

For this calculation, we should specify the reference frame in which we work, because the helicity amplitudes are frame dependent. This frame dependence is then eliminated with use of the invariant amplitudes. For the calculation, the Breit frame will be used. ${ }^{2}$ It is defined by $\vec{p}+\vec{p}^{\prime}=0$. Furthermore,

$$
\begin{aligned}
P^{\mu} & =\left(\sqrt{M^{2}-\frac{t}{4}}, \overrightarrow{0}\right), \\
\tilde{K}^{\mu} & =(\omega, 0,0, q), \\
L^{\mu} & =\left(0, \frac{\Delta}{2}, 0,0\right), \\
q_{1} & =\left(\omega, \frac{\Delta}{2}, 0, q\right), \\
q_{2} & =\left(\omega,-\frac{\Delta}{2}, 0, q\right),
\end{aligned}
$$

where $\Delta=\sqrt{-t}>0$. We next define the polarization vectors of the virtual photons with the photon helicity $\lambda_{1}= \pm 1,0$ referring to the initial photon and $\lambda_{2}= \pm 1,0$ to the final photon:

$$
\begin{aligned}
\varepsilon_{1}^{\mu}\left(\lambda_{1}= \pm 1\right) & =-\frac{1}{\sqrt{2}}\left(0, \frac{q}{q_{1}}, i \lambda_{1},-\frac{\Delta}{2 q_{1}}\right), \\
\varepsilon_{1}^{\mu}\left(\lambda_{1}=0\right) & =\frac{1}{\sqrt{Q^{2}}}\left(q_{1}, \frac{\omega}{q_{1}} \vec{q}_{1}\right),
\end{aligned}
$$

\footnotetext{
${ }^{2}$ Note that the $\gamma^{*} p$ c.m. frame cannot be used here, because in this frame it is impossible to fix the electrons' kinematics to the experimental one.
} 


$$
\begin{aligned}
\varepsilon_{2}^{\mu}\left(\lambda_{2}= \pm 1\right) & =-\frac{1}{\sqrt{2}}\left(0, \frac{q}{q_{1}}, i \lambda_{2}, \frac{\Delta}{2 q_{1}}\right), \\
\varepsilon_{2}^{\mu}\left(\lambda_{2}=0\right) & =\frac{1}{\sqrt{Q^{2}}}\left(q_{1}, \frac{\omega}{q_{1}} \vec{q}_{2}\right),
\end{aligned}
$$

where $q_{1}$ denotes the magnitude of the photon three-vector, $q_{1}=\left|\vec{q}_{1}\right|=\left|\vec{q}_{2}\right|=\sqrt{q^{2}-t / 4}$. The Compton helicity amplitudes are defined as

$$
T_{\lambda_{2} \lambda^{\prime} ; \lambda_{1} \lambda}=e^{2} \varepsilon_{1}^{\mu}\left(\lambda_{1}\right)\left(\varepsilon_{2}^{\nu}\left(\lambda_{2}\right)^{*} \cdot M_{\mu \nu}\right.
$$

where we explicitly took out the electric charge $e$. We next define shorthands,

$$
\begin{aligned}
& T_{\mathrm{LL}}=T_{0 \lambda^{\prime} ; 0 \lambda} \\
& T_{\mathrm{TT}}=T_{ \pm \lambda^{\prime} ; \pm \lambda} \\
& T_{\mathrm{TL}}=T_{ \pm \lambda^{\prime} ; 0 \lambda} \\
& T_{\mathrm{LT}}=T_{0 \lambda^{\prime} ; \pm \lambda} .
\end{aligned}
$$

With these definitions, we obtain:

$$
\begin{aligned}
& \frac{1}{e^{2}} T_{\mathrm{LL}}=\frac{4 Q^{2}}{t+4 Q^{2}} \bar{N}_{\lambda^{\prime}}^{\prime}\left[\frac{q^{2}}{q_{1}^{2}}\left(B_{1}+\tilde{K} B_{2}\right)-\frac{\omega^{2}}{q_{1}^{2}}\left(B_{17}+\tilde{K} B_{18}\right)\right. \\
& \left.-\frac{2 \omega}{\sqrt{M^{2}-\frac{t}{4}} q_{1}^{2}}\left(B_{9}+\tilde{K} B_{10}\right)\right] N_{\lambda} \text {, } \\
& \frac{1}{e^{2}} T_{\mathrm{TT}}=\frac{1}{2} \bar{N}_{\lambda^{\prime}}^{\prime}\left\{\frac{\omega^{2}}{q_{1}^{2}} \frac{t}{t+4 Q^{2}}\left(B_{1}+\tilde{K} B_{2}\right)\right. \\
& -\lambda_{1} \lambda_{2}\left(B_{3}+\tilde{K} B_{4}\right) \\
& +\frac{4 Q^{2}}{t+4 Q^{2}} \frac{2 \omega}{\sqrt{M^{2}-\frac{t}{4}} q_{1}^{2}}\left(B_{9}+\tilde{K} B_{10}\right) \\
& -\frac{16 Q^{4}}{t\left(t+4 Q^{2}\right)} \frac{q^{2}}{q_{1}^{2}}\left(B_{17}+\tilde{K} B_{18}\right) \\
& -\left(\lambda_{1}+\lambda_{2}\right) \frac{1}{\sqrt{M^{2}-\frac{t}{4}} q_{1}} i \not h \\
& \times\left[\frac{\omega}{\sqrt{M^{2}-\frac{t}{4}} q_{1}^{2}} B_{6}+\frac{16 Q^{2}}{t\left(t+4 Q^{2}\right)} B_{12}\right] \\
& +\left(\lambda_{2}-\lambda_{1}\right) \frac{1}{\sqrt{M^{2}-\frac{t}{4}} q_{1}} \gamma_{5} \\
& \left.\times\left[\frac{2 \omega}{\sqrt{M^{2}-\frac{t}{4}} q_{1}^{2}} B_{7}+\frac{16 Q^{2}}{t\left(t+4 Q^{2}\right)} B_{11}\right]\right\} N_{\lambda},
\end{aligned}
$$

$$
\begin{aligned}
\frac{1}{e^{2}} T_{\mathrm{TL}}= & -\frac{\sqrt{2 Q^{2}} \omega q}{\Delta q_{1}^{2}} \bar{N}_{\lambda^{\prime}}^{\prime}\left[\frac{t}{t+4 Q^{2}}\left(B_{1}+\tilde{K} B_{2}\right)\right. \\
& +\frac{4 Q^{2} q^{2}-t \omega^{2}}{q^{2}\left(t+4 Q^{2}\right)} \frac{1}{\sqrt{M^{2}-\frac{t}{4}} \omega}\left(B_{9}+\tilde{K} B_{10}\right)
\end{aligned}
$$

$$
\begin{aligned}
& \left.+\frac{4 Q^{2}}{t+4 Q^{2}} \frac{q^{2}}{q_{1}^{2}}\left(B_{17}+\tilde{K} B_{18}\right)\right] N_{\lambda}, \\
& -\frac{\sqrt{2 Q^{2}} \lambda_{2}}{\left(M^{2}-\frac{t}{4}\right) \Delta q q_{1}} \bar{N}_{\lambda^{\prime}}^{\prime}\left(\gamma_{5} B_{7}-i \not h B_{6}\right) N_{\lambda} \\
& +\frac{4 \sqrt{2 Q^{2}} \omega \lambda_{2} \bar{N}_{\lambda^{\prime}}^{\prime}\left(\gamma_{5} B_{11}-i \not h B_{12}\right) N_{\lambda}}{\sqrt{M^{2}-\frac{t}{4}} \Delta q q_{1}\left(t+4 Q^{2}\right)},
\end{aligned}
$$

$$
\begin{aligned}
\frac{1}{e^{2}} T_{\mathrm{LT}}= & \frac{\sqrt{2 Q^{2}} \omega q}{\Delta q_{1}^{2}} \bar{N}_{\lambda^{\prime}}^{\prime}\left[\frac{t}{t+4 Q^{2}}\left(B_{1}+\tilde{K} B_{2}\right)\right. \\
& +\frac{4 Q^{2} q^{2}-t \omega^{2}}{q^{2}\left(t+4 Q^{2}\right)} \frac{1}{\sqrt{M^{2}-\frac{t}{4}} \omega}\left(B_{9}+\tilde{K} B_{10}\right) \\
& \left.+\frac{4 Q^{2}}{t+4 Q^{2}} \frac{q^{2}}{q_{1}^{2}}\left(B_{17}+\tilde{K} B_{18}\right)\right] N_{\lambda}, \\
& -\frac{\sqrt{2 Q^{2}} \lambda_{1}}{\left(M^{2}-\frac{t}{4}\right) \Delta q q_{1}} \bar{N}_{\lambda^{\prime}}^{\prime}\left(\gamma_{5} B_{7}+i \not h B_{6}\right) N_{\lambda} \\
& +\frac{4 \sqrt{2 Q^{2}} \omega \lambda_{1}}{\sqrt{M^{2}-\frac{t}{4}} \Delta q q_{1}\left(t+4 Q^{2}\right)} \bar{N}_{\lambda^{\prime}}^{\prime}\left(\gamma_{5} B_{11}+i \not h B_{12}\right) N_{\lambda} .
\end{aligned}
$$

We next note that the amplitudes $B_{7}$ and $B_{11}$ do not contribute to the beam asymmetry. As can be seen from Eq. (38), the amplitudes $B_{6}$ and $B_{12}$ depend linearly on the photon helicities and therefore drop out of the "crosssection combination" of the helicity amplitudes, $\left(T_{1 \lambda^{\prime} ; 1 \lambda}+\right.$ $\left.T_{-1 \lambda^{\prime} ;-1 \lambda}\right)$. Instead, they obtain their contribution from the GDH sum rulelike combination $\left(T_{1 \lambda^{\prime} ; 1 \lambda}-T_{-1 \lambda^{\prime} ;-1 \lambda}\right)$, which vanishes in the considered kinematical regime according to Pomeranchuk's theorem. In previous work, the contribution of the photon helicity-flip Compton amplitudes was studied for the beam asymmetry $[15,16]$. In spite of double-logarithmic enhancement because of the quasi-RCS kinematics their contribution to the asymmetry $B_{n}$ was found to be negligibly small for forward kinematics and is neglected in the following.

The longitudinal-transverse amplitudes are in general nonzero. However, it is only the combination $T_{\mathrm{TL}}-T_{\mathrm{LT}}$, with the conserved photon helicity $\lambda_{1}=\lambda_{2}$, that contributes to the amplitudes $B_{1,2,3,4,9,10,17,18}$ relevant for the asymmetry. As a microscopic calculation shows (see, for instance, Ref. [17]) this difference vanishes for diffractive kinematics. Thus, the only nonzero helicity combinations are $\Sigma_{\mathrm{TT}} \equiv \frac{1}{2}\left(T_{1 \lambda^{\prime} ; 1 \lambda}+\right.$ $T_{-1 \lambda^{\prime} ;-1 \lambda}$ ) and $T_{\mathrm{LL}}$. Their imaginary parts are related to the transverse and longitudinal cross sections, respectively, which contain all the physical information on forward doubly virtual Compton scattering with an unpolarized target. For the remaining amplitudes $B_{i}$, we define shorthands, for example, $\mathbf{B}_{1}$ for $\bar{N}_{\lambda^{\prime}}^{\prime}\left(B_{1}+\widetilde{K} B_{2}\right) N_{\lambda}$, and find the expressions as functions of the helicity amplitudes:

$$
\begin{aligned}
e^{2} \mathbf{B}_{1} & =\frac{\omega^{2}}{q_{1}^{2}} \frac{t}{t+4 Q^{2}} \Sigma_{\mathrm{TT}}+\frac{q^{2}}{q_{1}^{2}} \frac{4 Q^{2}}{t+4 Q^{2}} T_{\mathrm{LL}}, \\
e^{2} \mathbf{B}_{\mathbf{3}} & =-\Sigma_{\mathrm{TT}},
\end{aligned}
$$




$$
\begin{aligned}
e^{2} \mathbf{B}_{9} & =(P \tilde{K}) \frac{q^{2}}{q_{1}^{2}} \frac{t}{t+4 Q^{2}}\left(\Sigma_{\mathrm{TT}}-T_{\mathrm{LL}}\right), \\
e^{2} \frac{4 Q^{2}}{t} \mathbf{B}_{17} & =-\frac{q^{2}}{q_{1}^{2}} \frac{4 Q^{2}}{t+4 Q^{2}} \Sigma_{\mathrm{TT}}-\frac{\omega^{2}}{q_{1}^{2}} \frac{t}{t+4 Q^{2}} T_{\mathrm{LL}} .
\end{aligned}
$$

Working out the spinors, we get in the Breit frame:

$$
\begin{aligned}
\bar{N}^{\prime} N & =2 \sqrt{M^{2}-\frac{t}{4}} 2 \lambda \delta_{\lambda-\lambda^{\prime}}, \\
\bar{N}^{\prime} \tilde{K} N & =2 M \omega 2 \lambda \delta_{\lambda-\lambda^{\prime}}+q \Delta 2 \lambda \delta_{\lambda \lambda^{\prime}} .
\end{aligned}
$$

We see that in the forward limit $t=0$, only nucleon helicity-flip amplitudes survive. This should not mislead the reader because the reason for this helicity flipping is the use of Breit frame kinematics for the nucleons: The initial and final nucleon three-momenta are opposite by definition; therefore, unchanged spin projection onto the $x$ axis will correspond to opposite helicities. Furthermore, the optical theorem relates the imaginary parts of the forward amplitudes to the corresponding cross sections,

$$
\begin{aligned}
\operatorname{Im} \frac{1}{2}\left(T_{1-\frac{1}{2} ; 1 \frac{1}{2}}+T_{-1-\frac{1}{2} ;-1 \frac{1}{2}}\right) & =2 \sqrt{M^{2}-t / 4} \omega \sigma_{T} \\
\operatorname{Im} T_{0-\frac{1}{2} ; 0 \frac{1}{2}} & =2 \sqrt{M^{2}-t / 4} \omega \sigma_{L} .
\end{aligned}
$$

If the Callan-Gross relation holds, the longitudinal cross section is related to the transvers one as $\sigma_{L}=Q^{2} / q_{1}^{2} \sigma_{T}$. Inserting these expressions into the tensor contraction of Eq. (28) and noting that $A b s M^{\mu \nu}=-2 \operatorname{Im} M^{\mu \nu}$, we obtain:

$$
\begin{aligned}
L_{\alpha \mu \nu} H_{\mathrm{EVVCS}}^{\alpha \mu \nu}= & -128 i m M G_{E} \omega(n \cdot \xi) \\
& \times\left[\frac{2 \omega E_{1}}{q^{2}}-\frac{Q^{2}}{q^{2}}-\frac{Q^{2}}{q_{1}^{2}}+\frac{Q^{4}}{q_{1}^{4}}\right] \frac{1}{e^{2}} \sigma_{T} \\
& +128 i m M^{3} L^{2} G_{E} \frac{\left(n k_{1}\right)\left(k_{1} \xi\right)}{n^{2}} \omega \frac{1}{e^{2}} \sigma_{T} .
\end{aligned}
$$

It can now be seen that spurious singularities $\sim 1 / t+4 Q^{2}$ appearing in the individual amplitudes in Eq. (41) cancel in the observable, as they should.

We next perform the integration over the electron phase space. We remind the reader that in the choosen kinematics

$$
\begin{aligned}
n^{\mu} & =\left(0,0,-\sqrt{M^{2}-\frac{t}{4}} q \frac{\Delta}{2}, 0\right), \\
\xi^{\mu} & =(0,0,1,0),
\end{aligned}
$$

therefore

$$
\begin{aligned}
\int \frac{\left|\vec{k}_{1}\right|^{2} d\left|\vec{k}_{1}\right| d \Omega_{k_{1}}}{2 E_{1}(2 \pi)^{3}} \frac{1}{Q_{1}^{2} Q_{2}^{2}} \operatorname{Im} L_{\alpha \mu \nu} H^{\alpha \mu \nu} \\
=-128 m M G_{E} \sqrt{M^{2}-\frac{t}{4} \frac{\Delta}{2} \frac{1}{e^{2}} \sigma_{T}} \\
\quad \times\left\{2 E I_{1}^{0}-2 J_{2}^{00}-2 I_{2}+I_{3}-\frac{2 M^{2}}{M^{2}-\frac{t}{4}} J_{25}\right\},
\end{aligned}
$$

where we have used the notation:

$$
\begin{aligned}
I_{1}^{\mu} & =\int \frac{\left|\vec{k}_{1}\right|^{2} d\left|\vec{k}_{1}\right| d \Omega_{k_{1}}}{2 E_{1}(2 \pi)^{3}} \frac{k_{1}^{\mu}}{Q_{1}^{2} Q_{2}^{2}} \\
I_{2} & =\int \frac{\left|\vec{k}_{1}\right|^{2} d\left|\vec{k}_{1}\right| d \Omega_{k_{1}}}{2 E_{1}(2 \pi)^{3}} \frac{1}{2}\left[\frac{1}{Q_{1}^{2}}+\frac{1}{Q_{2}^{2}}\right] \\
I_{3} & =\int \frac{\left|\vec{k}_{1}\right|^{2} d\left|\vec{k}_{1}\right| d \Omega_{k_{1}}}{2 E_{1}(2 \pi)^{3}} \frac{\left(Q_{1}^{2}+Q_{2}^{2}\right)^{2}}{4 Q_{1}^{2} Q_{2}^{2}} \frac{1}{\left(\vec{k}-\vec{k}_{1}\right)^{2}} \\
J_{2}^{\mu \nu} & =\int \frac{\left|\vec{k}_{1}\right|^{2} d\left|\vec{k}_{1}\right| d \Omega_{k_{1}}}{2 E_{1}(2 \pi)^{3}} \frac{k_{1}^{\mu} k_{1}^{\nu}}{Q_{1}^{2} Q_{2}^{2}} .
\end{aligned}
$$

Furthermore, the coefficient $J_{25}$ originates from decomposing the tensor integral according to [18],

$$
\begin{aligned}
J_{2}^{\mu \nu}= & J_{21} P^{\mu} P^{v}+J_{22} K^{\mu} K^{v}+J_{23}\left(P^{\mu} K^{v}+K^{\mu} P^{\nu}\right) \\
& +J_{24} L^{\mu} L^{\nu}+J_{25} g^{\mu \nu} .
\end{aligned}
$$

The integrals $I_{1}^{0}$ and $I_{2}$ entering Eq. (47) were calculated in the previous work [15]. We obtain for the first three integrals

$$
\begin{aligned}
I_{1}^{0}= & \frac{E}{-4 \pi^{2} t}\left\{\frac{E_{\mathrm{thr}}}{E} \ln \frac{\sqrt{-t}}{m} \frac{E_{\mathrm{thr}}}{E}\right. \\
& \left.+\left(1-\frac{E_{\mathrm{thr}}}{E}\right) \ln \left(1-\frac{E_{\mathrm{thr}}}{E}\right)\right\}, \\
I_{2}= & \frac{1}{4 \pi^{2}} \frac{E_{\mathrm{thr}}}{E} \ln \frac{2 E_{\mathrm{thr}}}{m} \\
& +\frac{1}{4 \pi^{2}}\left(1-\frac{E_{\mathrm{thr}}}{E}\right) \ln \left(1-\frac{E_{\mathrm{thr}}}{E}\right), \\
I_{3}= & \frac{1}{8 \pi^{2}}\left(1+\frac{E_{\mathrm{thr}}}{E}\right) \ln \left(1+\frac{E_{\mathrm{thr}}}{E}\right) \\
& +\frac{1}{8 \pi^{2}}\left(1-\frac{E_{\mathrm{thr}}}{E}\right) \ln \left(1-\frac{E_{\mathrm{thr}}}{E}\right),
\end{aligned}
$$

and for the tensor integrals:

$$
\begin{aligned}
J_{2}^{00}= & \frac{E_{\mathrm{thr}}^{2}}{-8 \pi^{2} t}\left[\ln \left(\frac{Q}{m} \frac{E_{\mathrm{thr}}}{E}\right)+\frac{E}{E_{\mathrm{thr}}}\right] \\
& +\frac{E^{2}}{-8 \pi^{2} t}\left(1-\frac{E_{\mathrm{thr}}^{2}}{E^{2}}\right) \ln \left(1-\frac{E_{\mathrm{thr}}}{E}\right) \\
J_{25}= & \frac{1}{32 \pi^{2}} \frac{E_{\mathrm{thr}}^{2}}{E^{2}+t / 4}\left[\ln \frac{\sqrt{-t}}{2 E}+\frac{E}{E_{\mathrm{thr}}}+\frac{1}{2}\right. \\
& \left.+\left(\frac{E^{2}}{E_{\mathrm{thr}}^{2}}-1\right) \ln \left(1-\frac{E_{\mathrm{thr}}}{E}\right)\right]-\frac{E_{\mathrm{thr}}^{2}}{32 \pi^{2}(p k)} .
\end{aligned}
$$

In the above formulas, $E_{\mathrm{thr}}$ denotes the upper limit in the integral over the electron energy, which corresponds to threshold production of the pion, $E_{\mathrm{thr}}=s-\left(M+m_{\pi}^{2}\right)^{2} / 2 \sqrt{M^{2}-t / 4}$ in the Breit frame. We refer the reader to the appendix for details of the calculation. We can finally write down the final result for normal beam spin asymmetry:

$$
\begin{aligned}
B_{n}= & -\frac{m \sqrt{-t} \sigma_{T}}{4 \pi^{2}(1+\tau)^{2}} \frac{G_{E}}{\tau G_{M}^{2}+\varepsilon G_{E}^{2}}\left[\ln \left(\frac{\sqrt{-t}}{m}\right)-1\right. \\
& \left.+\frac{t}{E^{2}} \ln \frac{E^{2}}{m^{2}}+\frac{t}{4 E^{2}}\left(\ln \frac{\sqrt{-t}}{2 E}+1+\frac{M^{2}}{2 s}\right)\right] .
\end{aligned}
$$




\section{RESULTS}

The model used in the previous section for the calculation of the $B_{n}$ is based on the optical theorem, Callan-Cross relation between the longitudinal and transverse partial cross sections, and the neglection of the mismatch between the virtualities of the virtual photons inside the loop. This latter assumption restricts the applicability of the above formula to low values of the momentum transfer. To estimate the error introduced by neglecting the terms $\sim\left(Q_{1}^{2}-Q_{2}^{2}\right)^{2} / Q_{1}^{2} Q_{2}^{2}$ under the integral over the electron phase space, we note that the integrals entering the final result of Eq. (52) have the order $\sim t^{-1}\left(I_{1}^{0}, J_{2}^{00}\right)$ and $t^{0}\left(I_{2}, I_{3}, J_{25}\right)$. As noted before, terms $\sim\left(Q_{1}^{2}-Q_{2}^{2}\right)^{2} /$ $Q_{1}^{2} Q_{2}^{2}$ contribute to the order $t^{1}$. Therefore, they contribute at relative order of $t^{2}$. Thus, for low values of $t$, our full result of Eq. (52) is consistent with the approximation used.

We next study the leading $t$ dependence of the beam normal spin asymmetry. It is given by

$$
B_{n}=-\frac{m \sqrt{-t} \sigma_{T}}{4 \pi^{2}} \frac{G_{E}}{\tau G_{M}^{2}+\varepsilon G_{E}^{2}}\left[\ln \left(\frac{\sqrt{-t}}{m}\right)-1\right] .
$$

This result is in exact agreement with the result of Ref. [14]. The approach of Ref. [14] consisted of taking only one amplitude for elastic doubly VCS, the one that survives in the exact forward RCS. It therefore amounts to neglecting not only the additional $t$ dependence of the Compton amplitude but also the dependence on the photon virtualities. The present calculation, on the contrary, uses the complete set of invariant amplitudes for the case of equal virtualities of the incoming and outgoing photons and does not a priori set any kinematical variable to zero. The reason for this agreement is that every factor $\sim Q_{1,2}^{2}$ in the numerator actually leads to an extra suppression in $t$, as can be seen, if comparing the integrals $I_{2}$ to $I_{0}$ and $B_{1}^{\mu}$ to $I_{1}^{\mu}$, respectively. The integrals are given in the appendix.

If using as input the exact forward value of the imaginary part of the Compton amplitude, to go to finite values of $t$, one needs a phenomenological information to consistently describe the off-forward Compton scattering. We use the same approach as in Ref. [14]. It is based on the phenomenological exponential fit of the $t$ dependence of the Compton differential cross section for values of $t$ from 0 through $-0.8 \mathrm{GeV}^{2}$. Because $d \sigma / d t \sim \sigma_{T}^{2}$, one gets

$$
\sigma_{T}(t)=\sigma_{T}(0) e^{B t / 2}
$$

with $B \approx 5.2-8.6 \mathrm{GeV}^{-2}$. For the energy dependence of $\sigma_{T}$, we use the phenomenological fit of Ref. [19]. We present our results in Fig. 1.

As seen from the figure, the more accurate account on the $t$ dependence leads to faster decreasing of the asymmetry with increasing $Q^{2}$. We predict therefore lower negative values for $B_{n}$ than the authors of Ref. [14].

In Fig. 2, results for $B_{n}$ on the neutron target are shown. The asymmetry is only about twice smaller for the neutron than for the proton, which is because the overall factor of $G_{E} / \tau G_{M}^{2}+\varepsilon G_{E}^{2}$. Because also the cross section in the denominator is much smaller for the neutron target at low $Q^{2}$ than for the proton, it means that a measurement of the cross

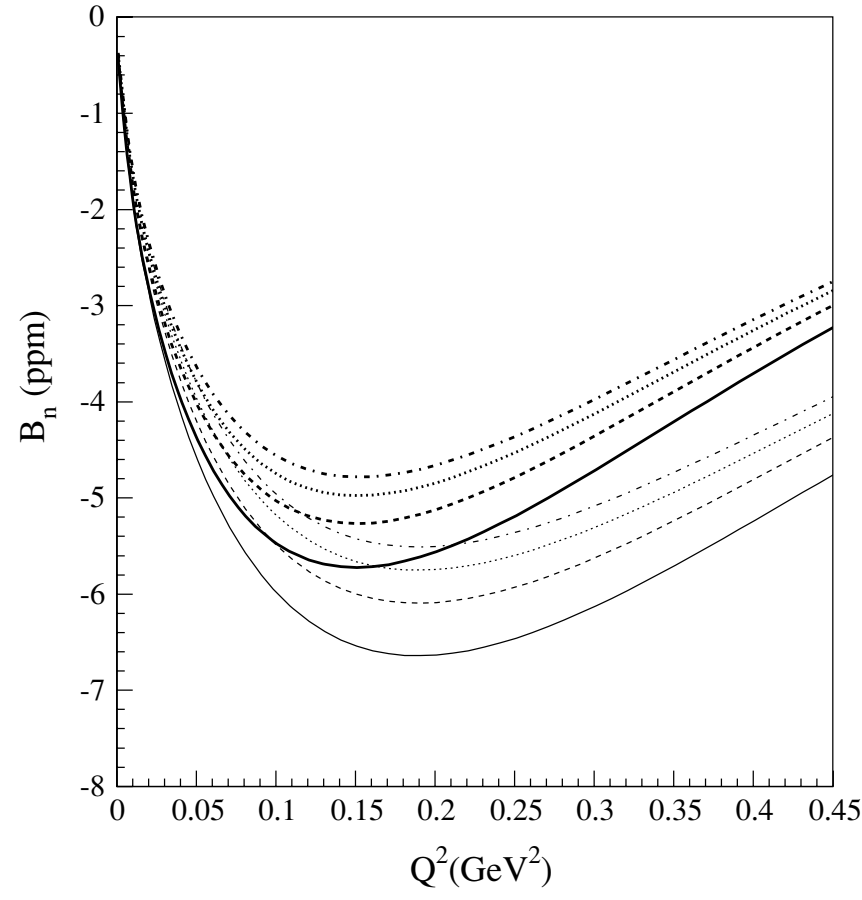

FIG. 1. The results for $B_{n}$ as function of the momentum transfer $Q^{2}$ are shown for four values of the lab beam energy: $3 \mathrm{GeV}$ (solid lines), $6 \mathrm{GeV}$ (dashed lines), $12 \mathrm{GeV}$ (dotted lines), and $45 \mathrm{GeV}$ (dash-dotted lines). The thick lines correspond to the full $t$ dependence of Eq. (52), whereas the thin lines correspond to the leading $t$ behavior of Eq. (53).

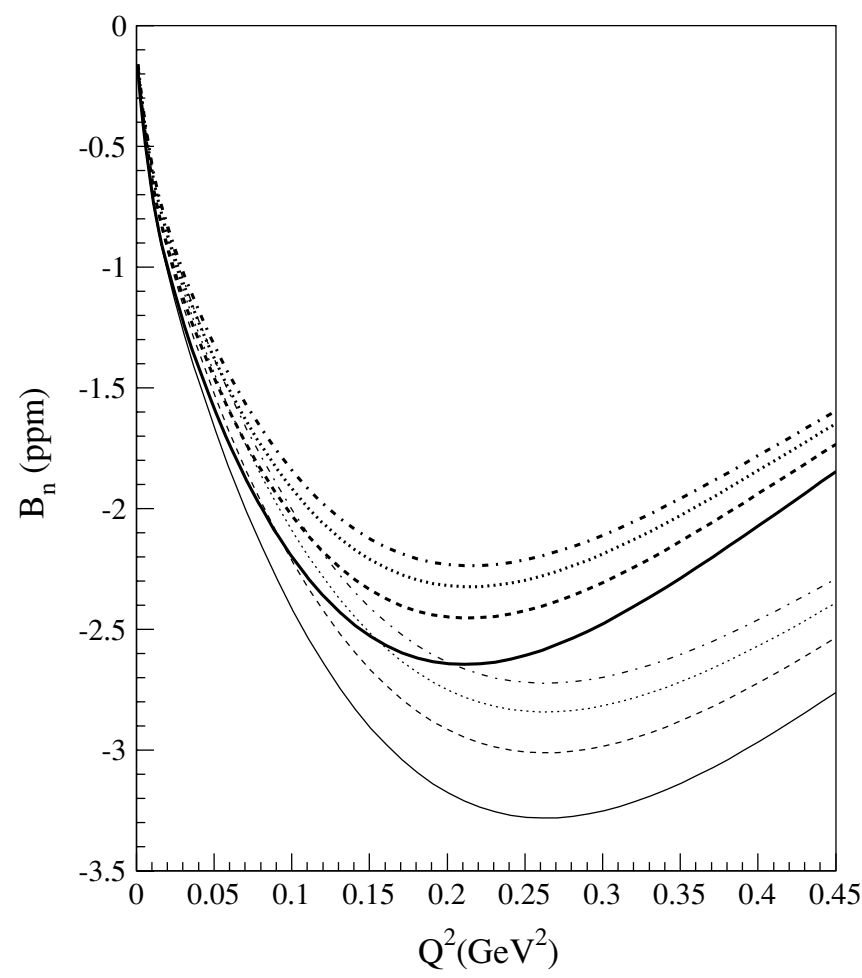

FIG. 2. The results for $B_{n}$ as function of the momentum transfer $Q^{2}$ for the neutron target. Notation as in Fig. 1 . 
section difference in the numerator of the $B_{n}$ would be a very challenging task for the neutron target.

\section{SUMMARY}

In summary, we constructed the invariant basis for VVCS. In the general case, VVCS is described by means of 18 independent invariant amplitudes that are functions of the invariants $P \tilde{K}, t, q_{1}^{2}, q_{2}^{2}$. The proposed form of the VVCS tensor does not exist in the literature. This form is based on Lorentz and gauge invariance and consists of constructing nine orthogonal basis tensors, which are useful for practical calculations. We furthermore investigated the behavior of the VVCS amplitudes under photon and nucleon crossing. Basing on this crossing behavior, it is possible to obtain the correct form of the VVCS basis in the case of elastic VVCS (i.e., $q_{1}^{2}=q_{2}^{2}$ ) where only 12 amplitudes survive. The special case of elastic VVCS is realized in the case of the calculation of the beam normal spin asymmetry $B_{n}$ with forward kinematics. We provide the calculation for this observable with these kinematics. Because in the exact forward limit this observable vanishes, one has to use the full amplitude for this calculation. We project the Compton helicity amplitudes onto the invariant basis and use as input the optical theorem. It relates the imaginary part of the helicity amplitudes $\frac{1}{2}\left(T_{1 \frac{1}{2}, 1 \frac{1}{2}}+T_{-1 \frac{1}{2},-1 \frac{1}{2}}\right)$ and $T_{0 \frac{1}{2}, 0 \frac{1}{2}}$ to the transverse $\sigma_{T}$ and the longitudinal $\sigma_{L}$ cross sections, respectively. The longitudinal cross section is then related to the transverse one by means of the Callan-Cross relation. The resulting asymmetry is of the order of $\approx-5 \mathrm{ppm}$ and is practically independent of the electron energy. The presented formalism may be furthermore extended to the two-photon exchange contributions to other observables.

\section{ACKNOWLEDGMENTS}

The author is grateful to Dr. Nicole F. Bell for useful comments on the manuscript. This work was supported by the US Department of Energy contract DOE-FG02-05ER 41361.

\section{APPENDIX}

\section{Projection technique}

In a real calculation, one needs to calculate the singleamplitude contributions from, for instance, a Feynman diagram. For this, one can make use of the fact that all the basis tensors are orthogonal. For example,

$$
\frac{P^{\prime \mu} P^{\prime \nu}}{P^{\prime 2}} W_{\mu \nu}=\bar{N}^{\prime}\left[B_{1}+\tilde{K} B_{2}\right] N
$$

and so on. We find following useful relations:

$$
\begin{aligned}
P^{\prime 2} & =P^{2}-\frac{P \tilde{K}}{\tilde{K}^{2}} \frac{L^{2}}{L^{\prime 2}} \\
L^{\prime 2} & =L^{2}-\frac{(L \tilde{K})^{2}}{\tilde{K}^{2}} \\
n^{2} & =-P^{\prime 2} \tilde{K}^{2} L^{\prime 2} \\
\tilde{K}^{\prime 2} & =-\frac{\tilde{K}^{2}}{L^{\prime 2}} Q_{1}^{2}
\end{aligned}
$$

$$
\begin{aligned}
\tilde{K}^{\prime \prime 2} & =-\frac{\tilde{K}^{2}}{L^{\prime 2}} Q_{2}^{2} \\
\tilde{K}^{\prime} \cdot \tilde{K}^{\prime \prime} & =\frac{\tilde{K}^{2}}{L^{\prime 2}}\left(L^{2}-\tilde{K}^{2}\right),
\end{aligned}
$$

and furthermore,

$$
\begin{aligned}
P^{\prime} \cdot \tilde{K} & =P^{\prime} \cdot L^{\prime}=P^{\prime} \cdot L=P^{\prime} \cdot \tilde{K}^{\prime}=P^{\prime} \cdot \tilde{K}^{\prime \prime}=P^{\prime} \cdot n \\
& =n \cdot \tilde{K}=n \cdot L^{\prime}=n \cdot L=n \cdot \tilde{K}^{\prime}=n \cdot \tilde{K}^{\prime \prime}=0 ; \\
q_{1} \cdot L^{\prime} & =-q_{2} \cdot L^{\prime}=L \cdot L^{\prime}=L^{\prime 2} .
\end{aligned}
$$

\section{Leptonic and hadronic tensors}

The hadronic tensors quoted in Eq. (25) are given by

$$
\begin{aligned}
A^{\mu \nu}= & \frac{P^{\prime \mu} P^{\prime \nu}}{P^{\prime 2}} B_{1}+\frac{n^{\mu} n^{\nu}}{n^{2}} B_{3}+\frac{\tilde{K}^{\prime \mu} P^{\prime \nu}}{P^{\prime 2} \tilde{K}^{2}} B_{9} \\
& +\frac{P^{\prime \mu} \tilde{K}^{\prime \prime \nu}}{P^{\prime 2} \tilde{K}^{2}} B_{13}+\frac{\tilde{K}^{\prime \mu} \tilde{K}^{\prime \prime \nu}}{\tilde{K}^{2}} B_{17} \\
B^{\mu \nu}= & \frac{P^{\prime \mu} P^{\prime \nu}}{P^{\prime 2}} B_{2}+\frac{n^{\mu} n^{\nu}}{n^{2}} B_{4}+\frac{\tilde{K}^{\prime \mu} P^{\prime \nu}}{P^{\prime 2} \tilde{K}^{2}} B_{10} \\
& +\frac{P^{\prime \mu} \tilde{K}^{\prime \prime \nu}}{P^{\prime 2} \tilde{K}^{2}} B_{14}+\frac{\tilde{K}^{\prime \mu} \tilde{K}^{\prime \prime \nu}}{\tilde{K}^{2}} B_{18} \\
C^{\mu \nu}= & \frac{P^{\prime \mu} n^{\nu}+n^{\mu} P^{\prime \nu}}{P^{\prime 2} n^{2}} B_{5}+\frac{P^{\prime \mu} n^{\nu}-n^{\mu} P^{\prime \nu}}{P^{\prime 2} n^{2}} B_{7} \\
+ & \frac{\tilde{K}^{\prime \mu} n^{\nu}}{n^{2} \tilde{K}^{2}} B_{11}+\frac{\tilde{K}^{\prime \prime \nu} n^{\mu}}{n^{2} \tilde{K}^{2}} B_{15} \\
D^{\mu \nu}= & \frac{P^{\prime \mu} n^{\nu}+n^{\mu} P^{\prime \nu}}{P^{\prime 2} n^{2}} B_{6}+\frac{P^{\prime \mu} n^{\nu}-n^{\mu} P^{\prime \nu}}{P^{\prime 2} n^{2}} B_{8} \\
+ & \frac{\tilde{K}^{\prime \mu} n^{\nu}}{n^{2} \tilde{K}^{2}} B_{12}+\frac{\tilde{K}^{\prime \prime \nu} n^{\mu}}{n^{2} \tilde{K}^{2}} B_{16} .
\end{aligned}
$$

The leptonic tensor

$$
L_{\alpha \mu \nu}=\operatorname{Tr}\left(k^{\prime}+m\right) \gamma_{\nu}\left(k_{1}+m\right) \gamma_{\mu} \gamma_{5} \sharp(k+m) \gamma_{\alpha}
$$

can be conveniently decomposed into two parts which are symmetric and antisymmetric in the pair of indices $(\mu \nu)$ :

$$
\begin{aligned}
L_{\alpha \mu \nu}^{\mathrm{SYM}}= & -8 i m\left\{g_{\mu \nu} \varepsilon_{\kappa \rho \tau \alpha} L^{\kappa} \tilde{K}^{\rho} \xi^{\tau}\right. \\
& \left.+\left(k_{1}\right)_{\mu} \varepsilon_{\kappa \nu \tau \alpha} L^{\kappa} \xi^{\tau}+\left(k_{1}\right)_{\nu} \varepsilon_{\kappa \mu \tau \alpha} L^{\kappa} \xi^{\tau}\right\}, \\
L_{\alpha \mu \nu}^{\mathrm{ASYM}}= & -8 i m\left\{\xi_{\nu} \varepsilon_{\kappa \mu \tau \alpha} L^{\kappa} K^{\tau}-\xi_{\mu} \varepsilon_{\kappa \nu \tau \alpha} L^{\kappa} K^{\tau}\right. \\
& +L_{\alpha} \varepsilon_{\nu \mu \tau \rho} \xi^{\tau} L^{\rho}-L^{2} \varepsilon_{\nu \mu \tau \alpha} \xi^{\tau} \\
& \left.-K_{\alpha} \varepsilon_{v \mu \tau \rho} \xi^{\tau} \tilde{K}^{\rho}+\xi_{\alpha} \varepsilon_{\nu \mu \tau \rho} k_{1}^{\tau} L^{\rho}\right\} .
\end{aligned}
$$

\section{Tensor contractions}

$$
\begin{aligned}
& {\left[L^{2} \tilde{K}^{\alpha}-(L \tilde{K}) L^{\alpha}\right] B^{\mu \nu} \cdot L_{\alpha \mu \nu}=-8 i m L^{2}(n \xi)} \\
& \quad \times\left\{\frac{2\left(P^{\prime} k_{1}\right)}{P^{\prime 2}} B_{2}+\frac{Q_{1}^{2}+Q_{2}^{2}}{4 n^{2}}\left[Q_{1}^{2} B_{10}+Q_{2}^{2} B_{14}\right]\right\} \\
& \quad+16 i m\left(L^{2}\right)^{2} \frac{\left(n k_{1}\right)(\tilde{K} \xi)}{n^{2}}(P \tilde{K}) B_{4} .
\end{aligned}
$$




$$
\begin{aligned}
& n^{\alpha} D^{\mu \nu} \cdot L_{\alpha \mu \nu}=16 i m \frac{\left(n k_{1}\right)(\tilde{K} \xi)}{n^{2}}\left\{-L^{2} B_{6}+(L \tilde{K}) B_{8}\right. \\
& \left.+\frac{(P \tilde{K}) L^{2}}{\tilde{K}^{2} L^{\prime 2}}\left[Q_{1}^{2} B_{12}+Q_{2}^{2} B_{16}\right]\right\} \text {. } \\
& P^{\alpha} A^{\mu \nu} \cdot L_{\alpha \mu \nu}=16 i m \frac{\left(n k_{1}\right)(\tilde{K} \xi)}{n^{2}} P^{2} L^{2} B_{3} \\
& +8 i m(n \xi)\left(B_{1}+B_{3}+\frac{2\left(P^{\prime} k_{1}\right)(P \tilde{K})}{n^{2}} B_{1}\right. \\
& +\left[\frac{\left(\tilde{K}^{\prime} \tilde{K}^{\prime \prime}\right)}{\tilde{K}^{2}}+\frac{2 K^{2}}{L^{\prime 2}}\right. \\
& \left.+\frac{\left(q_{1} L\right)\left(\tilde{K}^{\prime \prime} k_{1}\right)-\left(q_{2} L\right)\left(\tilde{K}^{\prime} k_{1}\right)}{\tilde{K}^{2} L^{\prime 2}}\right] B_{17} \\
& +\left\{\frac{(P \tilde{K}) L^{2}}{n^{2}}\left[\frac{\left(\tilde{K}^{\prime} k_{1}\right)}{\tilde{K}^{2}}-1\right]\right. \\
& \left.-\frac{(P K)\left(q_{1} \tilde{K}\right)+\left(P^{\prime} k_{1}\right)\left(q_{1} L\right)}{n^{2}}\right\} B_{10} \\
& +\left\{\frac{(P \tilde{K}) L^{2}}{n^{2}}\left[\frac{\left(\tilde{K}^{\prime \prime} k_{1}\right)}{\tilde{K}^{2}}-1\right]\right. \\
& \left.\left.-\frac{(P K)\left(q_{2} \tilde{K}\right)-\left(P^{\prime} k_{1}\right)\left(q_{2} L\right)}{n^{2}}\right\} B_{14}\right) \\
& =16 i m \frac{\left(n k_{1}\right)(\tilde{K} \xi)}{n^{2}} P^{2} L^{2} B_{3}+8 i m(n \xi)\left\{B_{1}\right. \\
& +B_{3}+\frac{2\left(P^{\prime} k_{1}\right)(P \tilde{K})}{n^{2}} B_{1}-\frac{L^{2} Q_{1}^{2} Q_{2}^{2}}{\tilde{K}^{2}\left(L^{\prime 2}\right)^{2}} B_{17} \\
& +\frac{Q_{1}^{2}}{n^{2}}\left[(P K)-(P \tilde{K}) \frac{\left(q_{2} L\right) L^{2}}{\tilde{K}^{2} L^{\prime 2}}\right] B_{9} \\
& \left.+\frac{Q_{2}^{2}}{n^{2}}\left[(P K)+(P \tilde{K}) \frac{\left(q_{1} L\right) L^{2}}{\tilde{K}^{2} L^{\prime 2}}\right] B_{13}\right\} \text {. }
\end{aligned}
$$

\section{Integrals over the electron phase space}

The integrals appearing in the final result are:

$$
\begin{aligned}
I_{0} & =\int \frac{\left|\vec{k}_{1}\right|^{2} d\left|\vec{k}_{1}\right| d \Omega_{k_{1}}}{2 E_{1}(2 \pi)^{3}} \frac{1}{Q_{1}^{2} Q_{2}^{2}} \\
I_{1}^{\mu} & =\int \frac{\left|\vec{k}_{1}\right|^{2} d\left|\vec{k}_{1}\right| d \Omega_{k_{1}}}{2 E_{1}(2 \pi)^{3}} \frac{k_{1}^{\mu}}{Q_{1}^{2} Q_{2}^{2}} \\
I_{2} & =\int \frac{\left|\vec{k}_{1}\right|^{2} d\left|\vec{k}_{1}\right| d \Omega_{k_{1}}}{2 E_{1}(2 \pi)^{3}} \frac{1}{2}\left[\frac{1}{Q_{1}^{2}}+\frac{1}{Q_{2}^{2}}\right] \\
I_{3} & =\int \frac{\left|\vec{k}_{1}\right|^{2} d\left|\vec{k}_{1}\right| d \Omega_{k_{1}}}{2 E_{1}(2 \pi)^{3}} \frac{\left(Q_{1}^{2}+Q_{2}^{2}\right)^{2}}{4 Q_{1}^{2} Q_{2}^{2}} \frac{1}{\left(\vec{k}-\vec{k}_{1}\right)^{2}} \\
J_{2}^{\mu \nu} & =\int \frac{\left|\vec{k}_{1}\right|^{2} d\left|\vec{k}_{1}\right| d \Omega_{k_{1}}}{2 E_{1}(2 \pi)^{3}} \frac{k_{1}^{\mu} k_{1}^{v}}{Q_{1}^{2} Q_{2}^{2}} .
\end{aligned}
$$

Furthermore, the terms $\sim\left(Q_{1}^{2}-Q_{2}^{2}\right)^{2} / Q_{1}^{2} Q_{2}^{2}$, which we have consistently neglected throughout the calculation, lead to the integral

$$
I_{4}=\int \frac{\left|\vec{k}_{1}\right|^{2} d\left|\vec{k}_{1}\right| d \Omega_{k_{1}}}{2 E_{1}(2 \pi)^{3}} \frac{\left(Q_{1}^{2}-Q_{2}^{2}\right)^{2}}{Q_{1}^{2} Q_{2}^{2}}=16 L_{\mu} L_{\nu} J_{2}^{\mu \nu} .
$$

The first integral was already calculated $[13,15]$. We next turn to the vector integral

$$
\begin{aligned}
I_{1}^{\mu} & =\int_{0}^{k_{\mathrm{thr}}} \frac{k_{1}^{2} d k_{1}}{2 E_{1}(2 \pi)^{3}} \int d \Omega_{k_{1}} \frac{k_{1}^{\mu}}{\left(k-k_{1}\right)^{2}\left(k^{\prime}-k_{1}\right)^{2}} \\
& =I_{1 P} P^{\mu}+I_{1 K} K^{\mu} .
\end{aligned}
$$

It cannot depend on $q^{\mu}$ because of the symmetry of $I_{1}$ under interchanging $k$ and $k^{\prime}$. To determine these two coefficients we have a system of equations,

$$
\begin{aligned}
K^{0} I_{1 K}+P^{0} I_{1 P} & =I_{1}^{0}=\frac{1}{16 \pi^{3}} \int \frac{d^{3} \vec{k}_{1}}{Q_{1}^{2} Q_{2}^{2}} \\
-t I_{1 K}+4 P K I_{1 P} & =4 K_{\mu} I_{1}^{\mu} \\
& =\int \frac{d^{3} \vec{k}_{1}}{(2 \pi)^{3} E_{1} Q_{1}^{2}} \equiv I_{2} .
\end{aligned}
$$

Using the same approach as for $I_{0}$ (see the appendix of Ref. [15]), we obtain for $I_{1}^{0}$ :

$$
\begin{aligned}
I_{1}^{0}= & \frac{E}{-8 \pi^{2} t} \int_{\frac{m}{E}}^{\frac{E_{\mathrm{thr}}}{E}} \frac{z d z}{\sqrt{z^{2}-\frac{m^{2}}{E^{2}}-\frac{4 m^{2}}{t}(1-z)^{2}}} \\
& \times \ln \frac{\sqrt{z^{2}-\frac{m^{2}}{E^{2}}-\frac{4 m^{2}}{t}(1-z)^{2}}+\sqrt{z^{2}-\frac{m^{2}}{E^{2}}}}{\sqrt{z^{2}-\frac{m^{2}}{E^{2}}-\frac{4 m^{2}}{t}(1-z)^{2}}-\sqrt{z^{2}-\frac{m^{2}}{E^{2}}}} \\
= & \frac{E}{-4 \pi^{2} t}\left[\frac{E_{\mathrm{thr}}}{E} \ln \frac{\sqrt{-t}}{m} \frac{E_{\mathrm{thr}}}{E}\right. \\
& \left.+\left(1-\frac{E_{\mathrm{thr}}}{E}\right) \ln \left(1-\frac{E_{\mathrm{thr}}}{E}\right)\right] .
\end{aligned}
$$

Finally, we consider the integral $I_{2}$ where we perform the angular integration,

$$
\begin{aligned}
I_{2} & =\frac{1}{(2 \pi)^{3}} \int \frac{d^{3} \vec{k}_{1}}{E_{1} Q_{1}^{2}} \\
& =\frac{1}{8 \pi^{2}} \int_{\frac{m}{E}}^{\frac{E_{\mathrm{thr}}}{E}} d z \ln \frac{z-\frac{m^{2}}{E^{2}}+\sqrt{1-\frac{m^{2}}{E^{2}}} \sqrt{z^{2}-\frac{m^{2}}{E^{2}}}}{z-\frac{m^{2}}{E^{2}}-\sqrt{1-\frac{m^{2}}{E^{2}}} \sqrt{z^{2}-\frac{m^{2}}{E^{2}}}} .
\end{aligned}
$$

After integrating by parts and changing variables, $z=$ $\frac{m}{E} \cosh y$ and $y=\ln t$, we arrive at

$$
\begin{aligned}
I_{2}= & \frac{1}{4 \pi^{2}} \frac{E_{\mathrm{thr}}}{E} \ln \frac{2 E_{\mathrm{thr}}}{m} \\
& +\frac{1}{4 \pi^{2}}\left(1-\frac{E_{\mathrm{thr}}}{E}\right) \ln \left(1-\frac{E_{\mathrm{thr}}}{E}\right) .
\end{aligned}
$$

In these last two integrals, it is important to keep $E_{\mathrm{thr}} / E$ unequal to 1 until the end to ensure the convergence of 
the integral. Solving the system of linear equations for the coefficients, we obtain:

$$
\begin{aligned}
& I_{1 P}=\frac{1}{8 \pi^{2}} \frac{s-M^{2}}{M^{4}-s u} \frac{E_{\mathrm{thr}}}{E} \ln \frac{2 E}{Q} \\
& I_{1 K}=\frac{1}{Q^{2}} I_{2}-\frac{4 P K}{Q^{2}} I_{1 P} .
\end{aligned}
$$

We next consider the integral $I_{3}$ and rewrite it as

$$
I_{3}=\int \frac{\left|\vec{k}_{1}\right|^{2} d\left|\vec{k}_{1}\right| d \Omega_{k_{1}}}{2 E_{1}(2 \pi)^{3}}\left[1+\frac{\left(Q_{1}^{2}-Q_{2}^{2}\right)^{2}}{4 Q_{1}^{2} Q_{2}^{2}}\right] \frac{1}{\left(\vec{k}-\vec{k}_{1}\right)^{2}} \text {. }
$$

We note that the second term in the square brackets $\sim\left(Q_{1}^{2}-Q_{2}^{2}\right)^{2}$ is of the order $t / s$. For the moment, we keep this subleading term and calculate the leading in $t$ contribution:

$$
\begin{aligned}
I_{3}= & \int \frac{\left|\vec{k}_{1}\right|^{2} d\left|\vec{k}_{1}\right| d \Omega_{k_{1}}}{2 E_{1}(2 \pi)^{3}} \frac{1}{\left(\vec{k}-\vec{k}_{1}\right)^{2}} \\
= & \frac{1}{8 \pi^{2}} \int_{0}^{k_{M}} d z \ln \frac{1+z}{1-z}+O(t / s) \\
= & \frac{1}{8 \pi^{2}}\left(1+\frac{E_{\mathrm{thr}}}{E}\right) \ln \left(1+\frac{E_{\mathrm{thr}}}{E}\right) \\
& +\frac{1}{8 \pi^{2}}\left(1-\frac{E_{\mathrm{thr}}}{E}\right) \ln \left(1-\frac{E_{\mathrm{thr}}}{E}\right)+O(t / s) .
\end{aligned}
$$

As we see, the leading term contributes to $B_{n}$ to relative order $t^{1}$. This means that the second term should be omitted to be consistent with the approximation adopted.

The calculation of the tensor integral is more involved. We decompose this tensor into tensorial structures constructed out of external momenta [18],

$$
\begin{aligned}
J_{2}^{\mu \nu}= & \int \frac{\left|\vec{k}_{1}\right|^{2} d\left|\vec{k}_{1}\right| d \Omega_{k_{1}}}{2 E_{1}(2 \pi)^{3}} \frac{k_{1}^{\mu} k_{1}^{\nu}}{Q_{1}^{2} Q_{2}^{2}} \\
= & J_{21} P^{\mu} P^{v}+J_{22} K^{\mu} K^{v}+J_{23}\left(P^{\mu} K^{v}+P^{v} K^{\mu}\right) \\
& +J_{24} L^{\mu} L^{\nu}+J_{25} g^{\mu \nu} .
\end{aligned}
$$

The final result contains. To calculate the coefficients $J_{2 j}$, we contract the tensor integral with $g^{\mu \nu}$ and external momenta:

$$
\begin{aligned}
& J_{2 \mu}^{\mu}=P^{2} J_{21}+K^{2} J_{22}+2 P K J_{23}+L^{2} J_{24}+4 J_{25} \\
&=m^{2} \int \frac{\left|\vec{k}_{1}\right|^{2} d\left|\vec{k}_{1}\right| d \Omega_{k_{1}}}{2 E_{1}(2 \pi)^{3}} \frac{1}{Q_{1}^{2} Q_{2}^{2}} \approx 0 \\
& 2 k_{\mu} 2 k_{v}^{\prime} J_{2}^{\mu \nu}=(2 P K)^{2} J_{21}+4\left(K^{2}\right)^{2} J_{22} \\
&+8 K^{2} P K J_{23}-4\left(L^{2}\right)^{2} J_{24}+8 K^{2} J_{25} \\
&= \int \frac{\left|\vec{k}_{1}\right|^{2} d\left|\vec{k}_{1}\right| d \Omega_{k_{1}}}{2 E_{1}(2 \pi)^{3}} \equiv C_{0}=\frac{E_{\mathrm{thr}}^{2}}{8 \pi^{2}} \\
& 2 k_{v}^{\prime} J_{2}^{\mu \nu}= 2 P K J_{21} P^{\mu}+2 K^{2} J_{22} K^{\mu} \\
&+2\left[P K K^{\mu}+K^{2} P^{\mu}\right] J_{23} \\
&-2 L^{2} J_{24} L^{\mu}+2 J_{25}(K-L)^{\mu} \\
&= \int \frac{\left|\vec{k}_{1}\right|^{2} d\left|\vec{k}_{1}\right| d \Omega_{k_{1}}}{2 E_{1}(2 \pi)^{3}} \frac{k_{1}^{\mu}}{Q_{1}^{2}} \equiv B_{1}^{\mu} .
\end{aligned}
$$

Rewriting the vector integral $B_{1}$ as

$$
\begin{aligned}
B_{1}^{\mu} & =\int \frac{\left|\vec{k}_{1}\right|^{2} d\left|\vec{k}_{1}\right| d \Omega_{k_{1}}}{2 E_{1}(2 \pi)^{3}} \frac{k_{1}^{\mu}}{Q_{1}^{2}}=B_{11} p^{\mu}+B_{12} k^{\mu} \\
& =B_{11} P^{\mu}+B_{12} K^{\mu}+\left(B_{12}-B_{11}\right) L^{\mu}
\end{aligned}
$$

we find following relations between $J_{2 i}$ and $B_{i}$ :

$$
\begin{aligned}
2 P K J_{21}+2 K^{2} J_{23} & =B_{11} \\
2 K^{2} J_{22}+2 P K J_{23}+2 J_{25} & =B_{12} \\
2 L^{2} J_{24}+2 J_{25} & =B_{11}-B_{12} .
\end{aligned}
$$

To proceed, we calculate the appearing vector integral. The coefficients can be found as following:

$$
2 k_{\mu} B_{1}^{\mu}=2 p k B_{11}=C_{0},
$$

whereas the 0 -component $B_{1}^{0}=p^{0} B_{11}+k^{0} B_{12}$ can be calculated directly:

$$
\begin{aligned}
B_{1}^{0} & =\int \frac{\left|\vec{k}_{1}\right|^{2} d\left|\vec{k}_{1}\right| d \Omega_{k_{1}}}{16 \pi^{3}} \frac{1}{Q_{1}^{2}} \\
& =\frac{E}{16 \pi^{2}} \int_{\frac{m}{E}}^{\frac{E_{\mathrm{thr}}}{E}} z d z \ln \frac{z+\sqrt{z^{2}-\frac{m^{2}}{E^{2}}}}{z-\sqrt{z^{2}-\frac{m^{2}}{E^{2}}}} \\
& =\frac{E_{\mathrm{thr}}^{2}}{16 \pi^{2} E}\left[\ln \frac{2 E_{\mathrm{thr}}}{m}-\frac{1}{2}\right] .
\end{aligned}
$$

The fifth independant equation for five unknown coefficients $J_{2 j}$ is obtained by the calculation of the $J_{2}^{00}$ component of the tensor integral. The calculation follows the same steps as that for $I_{1}^{0}$ given in the appendix of Ref. [15]. We obtain:

$$
\begin{aligned}
J_{2}^{00}= & \int \frac{\left|\vec{k}_{1}\right|^{2} d\left|\vec{k}_{1}\right| d \Omega_{k_{1}}}{16 \pi^{3}} E_{1} \frac{1}{Q_{1}^{2} Q_{2}^{2}} \\
= & \frac{E^{2}}{-8 \pi^{2} t} \int_{0}^{\frac{k_{\text {thr }}}{k}} \frac{z^{2} d z}{\sqrt{z^{2}-\frac{4 m^{2}}{t}(1-z)^{2}}} \\
& \times \ln \frac{z+\sqrt{z^{2}-\frac{4 m^{2}}{t}(1-z)^{2}}}{\sqrt{z^{2}-\frac{4 m^{2}}{t}(1-z)^{2}}-z} .
\end{aligned}
$$

Because of two powers of $z=k_{1} / k$ in the numerator, the contribution of small values of the intermediate electron momenta is negligible, and the square root can be Taylorexpanded because $m^{2} /|t| \ll 1$. This leads to

$$
\begin{aligned}
J_{2}^{00}= & \frac{E^{2}}{-8 \pi^{2} t} \int_{0}^{\frac{E_{\mathrm{thr}}}{E}} z d z \ln \left[\frac{-t}{m^{2}} \frac{z^{2}}{(1-z)^{2}}\right] \\
= & \frac{E_{\mathrm{thr}}^{2}}{-8 \pi^{2} t}\left[\ln \frac{\sqrt{-t} E_{\mathrm{thr}}}{m E}+\frac{E}{E_{\mathrm{thr}}}\right. \\
& \left.+\frac{E^{2}}{E_{\mathrm{thr}}^{2}}\left(1-\frac{E_{\mathrm{thr}}^{2}}{E^{2}}\right) \ln \left(1-\frac{E_{\mathrm{thr}}}{E}\right)\right] .
\end{aligned}
$$


Solving the system of linear equations for the coefficients $J_{2 j}$, we find

$$
\begin{aligned}
J_{25}= & \frac{P^{2}}{P^{\prime 2}}\left(J_{2}^{00}-\frac{E}{2 K^{2}} B_{1}^{0}\right)-\frac{1}{4(p k)} C_{0} \\
= & \frac{1}{32 \pi^{2}} \frac{E_{\mathrm{thr}}^{2}}{E^{2}+t / 4}\left[\ln \frac{\sqrt{-t}}{2 E}+\frac{E}{E_{\mathrm{thr}}}+\frac{1}{2}\right. \\
& \left.+\left(\frac{E^{2}}{E_{\mathrm{thr}}^{2}}-1\right) \ln \left(1-\frac{E_{\mathrm{thr}}}{E}\right)\right]-\frac{E_{\mathrm{thr}}^{2}}{32 \pi^{2}(p k)} .
\end{aligned}
$$

In the latter equation, the vector $P^{\prime \mu}$ is defined as $P^{\prime \mu}=$ $P^{\mu}-P K / K^{2} K^{\mu}$ with $P^{\prime 2}=P^{2}-(P K)^{2} / K^{2}$, where $K^{\mu}=$ $\frac{1}{2}\left(k+k^{\prime}\right)^{\mu}$.

Finally, the integral $I_{4}$ is given by

$$
\begin{aligned}
I_{4} & =\int \frac{\left|\vec{k}_{1}\right|^{2} d\left|\vec{k}_{1}\right| d \Omega_{k_{1}}}{2 E_{1}(2 \pi)^{3}} \frac{\left(Q_{1}^{2}-Q_{2}^{2}\right)^{2}}{Q_{1}^{2} Q_{2}^{2}}=16 L_{\mu} L_{\nu} J_{2}^{\mu \nu} \\
& =8 L^{2}\left(2 L^{2} J_{24}+2 J_{25}\right)=2 t\left(B_{11}-B_{12}\right) \\
& =\frac{-t}{16 \pi^{2}} \frac{E_{\mathrm{thr}}^{2}}{E^{2}}\left[\ln \left(\frac{4 E_{\mathrm{thr}}^{2}}{m^{2}}\right)-3\right] .
\end{aligned}
$$

[1] M. K. Jones et al., Phys. Rev. Lett. 84, 1398 (2000); O. Gayou et al., ibid. 88, 092301 (2002); V. Punjabi et al., Phys. Rev. C 71, 055202 (2005); Erratum-ibid. 71, 069902 (2005).

[2] L. Andivahis et al., Phys. Rev. D 50, 5491 (1994); M. E. Christy et al., Phys. Rev. C 70, 015206 (2004); I. A. Qattan et al., Phys. Rev. Lett. 94, 142301 (2005).

[3] P. A. M. Guichon and M. Vanderhaeghen, Phys. Rev. Lett. 91, 142303 (2003).

[4] P. G. Blunden, W. Melnitchouk, and J. A. Tjon, Phys. Rev. Lett. 91, 142304 (2003); P. G. Blunden, W. Melnitchouk, and J. A. Tjon, Phys. Rev. C 72, 034612 (2005).

[5] S. Kondratyuk, P. G. Blunden, W. Melnitchouk, and J. A. Tjon, Phys. Rev. Lett. 95, 172503 (2005).

[6] Y. C. Chen, A. Afanasev, S. J. Brodsky, C. E. Carlson, and M. Vanderhaeghen, Phys. Rev. Lett. 93, 122301 (2004).

[7] N. F. Mott, Proc. R. Soc. London, Ser. A135 429 (1935); A. O. Barut and C. Fronsdal, Phys. Rev. 120, 1871 (1960); A. De Rujula, J. M. Kaplan, and E. De Rafael, Nucl. Phys. B35, 365 (1971).

[8] S. P. Wells et al. (SAMPLE Collaboration), Phys. Rev. C 63 064001 (2001); F. Maas et al. (MAMI A4 Collaboration), Phys. Rev. Lett. 94, 082001 (2005); SLAC E158 Experiment, contact person K. Kumar; G. Cates, K. Kumar, and D. Lhuillier, spokespersons HAPPEX-2 Experiment, JLab
E-99-115; D. Beck, spokesperson JLab/G0 Experiment, JLab E-00-6, E-01-116.

[9] A. Afanasev, I. Akushevich, and N. P. Merenkov, [arXiv:hepph/0208260]; B. Pasquini and M. Vanderhaeghen, Phys. Rev. C 70, 045206 (2004); L. Diaconescu and M. J. Ramsey-Musolf, ibid. 70, 054003 (2004); M. Gorchtein, P. A. M. Guichon, and M. Vanderhaeghen, Nucl. Phys. A741, 234 (2004); A. V. Afanasev and N. P. Merenkov, Phys. Lett. B599, 48 (2004); Phys. Rev. D 70, 073002 (2004); [arXiv: hep-ph/0407167 v2]; M. Gorchtein, hep-ph/0505022; D. Borisyuk and A. Kobushkin, [arXiv: nucl-th/0508053].

[10] R. Tarrach, Nuovo Cimento A 28409 (1975).

[11] R. E. Prange, Phys. Rev. 110240 (1958).

[12] R. A. Berg and C. N. Lindner, Nucl. Phys. 26259 (1961).

[13] A. V. Afanasev and N. P. Merenkov, Phys. Lett. B599, 48 (2004); Phys. Rev. D 70, 073002 (2004).

[14] A. V. Afanasev and N. P. Merenkov, [arXiv: hep-ph/0407167 v2].

[15] M. Gorchtein, [arXiv: hep-ph/0505022].

[16] M. Gorchtein, submitted to Phys. Rev. C; [arXiv: hep$\mathrm{ph} / 0512105]$.

[17] S. J. Brodsky, P. Hoyer, and L. Magnea, Phys. Rev. D 55, 5585 (1997).

[18] G. Passarino and M. J. G. Veltman, Nucl. Phys. B160, 151 (1979).

[19] M. M. Block and F. Halzen, Phys. Rev. D 70, 091901(R) (2004). 